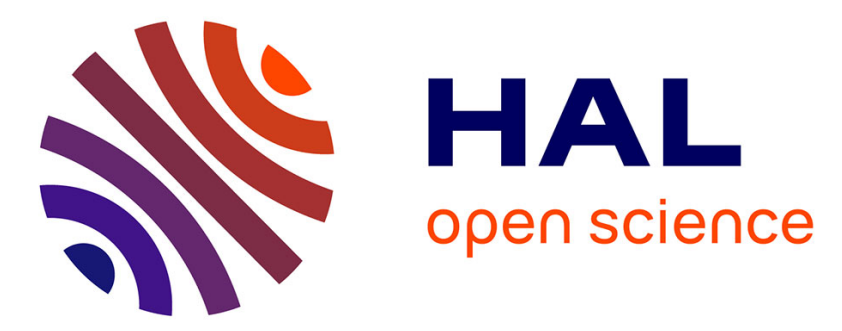

\title{
Vibrational Coupled Cluster Computations in Polyspherical Coordinates with the Exact Analytical Kinetic Energy Operator
}

\author{
Emil Lund Klinting, D. Lauvergnat, Ove Christiansen
}

\section{To cite this version:}

Emil Lund Klinting, D. Lauvergnat, Ove Christiansen. Vibrational Coupled Cluster Computations in Polyspherical Coordinates with the Exact Analytical Kinetic Energy Operator. Journal of Chemical Theory and Computation, 2020, 16 (7), pp.4505-4520. 10.1021/acs.jctc.0c00261 . hal-02994949

\author{
HAL Id: hal-02994949 \\ https://hal.science/hal-02994949
}

Submitted on 10 Nov 2020

HAL is a multi-disciplinary open access archive for the deposit and dissemination of scientific research documents, whether they are published or not. The documents may come from teaching and research institutions in France or abroad, or from public or private research centers.
L'archive ouverte pluridisciplinaire HAL, est destinée au dépôt et à la diffusion de documents scientifiques de niveau recherche, publiés ou non, émanant des établissements d'enseignement et de recherche français ou étrangers, des laboratoires publics ou privés. 


\title{
Vibrational Coupled Cluster Computations in Polyspherical Coordinates with the Exact Analytical Kinetic Energy Operator
}

\author{
Emil Lund Klinting, ${ }^{\dagger}$ David Lauvergnat, ${ }^{*, \ddagger}$ and Ove Christiansen*,† \\ $\dagger$ Department of Chemistry, Aarhus University, DK-8000 Aarhus C, Denmark \\ $\ddagger$ Université Paris-Saclay, CNRS, Institut de Chimie Physique, UMR8000, 91405 Orsay, \\ France
}

E-mail: david.lauvergnat@lcp.u-psud.fr; ove@chem.au.dk

\begin{abstract}
We present the first use of curvilinear vibrational coordinates, specifically polyspherical coordinates, in combination with vibrational coupled cluster theory. The polyspherical coordinates are used in the context of both the adaptive density-guided approach to potential energy surface construction and in the subsequent vibrational coupled cluster calculations of anharmonic vibrational states. Results obtained based on the polyspherical coordinate parametrization are compared to results obtained with the use of rectilinear vibrational coordinates, namely normal coordinates and hybrid optimized and localized coordinates for the formaldehyde molecule. This comparison is carried out with the full vibrational configuration interaction model, using the respective fully coupled potential energy surfaces and untruncated kinetic energy operators. The polyspherical coordinates are shown to facilitate an acceleration of convergence for truncated methods, when compared to the use of normal coordinates. We furthermore report on calculations on the hydrogen peroxide molecule in polyspherical coordinate
\end{abstract}


parametrization. The polyspherical vibrational coordinates are shown to perform very well, even for truncated methods, especially when considering the difficulty that rectilinear vibrational coordinates can exhibit in treating complicated internal molecular motion.

\section{Introduction}

The choice of vibrational coordinates is an important one in the context of solving the nuclear Schrödinger equation, as these will determine the functional form of the nuclear kinetic energy operator (KEO) and potential energy surface (PES). This choice can only be considered unimportant, when the exact vibrational wave function and full PES are used. Alas, truncation methods are often necessary, in order to ensure feasible computations and the choice of vibrational coordinates is therefore a contributing factor to the rate of convergence towards the exact limit for these methods. The first choice in choosing a set of vibrational coordinates, is to decide if these should be constructed as linear or nonlinear functions of Cartesian coordinates, which leads to rectilinear or curvilinear coordinates respectively. ${ }^{1}$ The former provides simpler equations for the nuclear KEO, while the latter more naturally account for large amplitude internal motion of the molecular system. The convergence of the PES and wave function expansions can, moreover, become increasingly slow with increasingly larger amplitude motions for rectilinear coordinates. ${ }^{2}$

The set of vibrational coordinates most widely used is probably the rectilinear normal coordinates (NCs), which can be expected to provide a reasonable good description of molecular vibrations, for small amplitude vibrational motions around the equilibrium geometry. ${ }^{3}$ The normal modes typically involve delocalized vibrational motion, often across the entire molecular system. This can needlessly complicate calculations, as artificial coupling between coordinates can be introduced and thereby prohibits truncation of the Hamilton operator at low levels of coupling, without loosing accuracy. ${ }^{1}$ This can become problematic, when incre-

mental truncation methods, like the $n$-mode expansion, ${ }^{4-15}$ are needed to construct molecular 
PESs. Suffice to say, it is generally a good strategy to employ vibrational coordinates that minimize couplings between different vibrational motions, especially if these vibrations are spatially far apart.

The idea of variationally optimizing the NCs have been around for quite some years now. ${ }^{16-18}$ The objective is to obtain a new set of coordinates that are linear combinations of the initial NCs but with a comparatively lesser degree of coupling between the modes. These optimized coordinates are generated via vibrational self-consistent field (VSCF) $)^{7,19-23}$ calculations and have been demonstrated to facilitate faster convergence towards the full vibrational configuration interaction (FVCI) limit, in vibrational structure calculations. ${ }^{18,24-29}$

A different approach to reduce the delocalization of NCs is to employ a localization scheme, which relies on geometrical localization criteria, resulting in localized coordinates. ${ }^{30-40}$ Localizing selected modes facilitates a faster convergence towards the FVCI limit for vibrational structure calculations and faster convergence in the n-mode expansion of the PES, as fewer high-order couplings are required.

The hybrid optimized and localized coordinates (HOLCs) are constructed with the use of both geometrical localization criteria and VSCF calculations, as a way of capturing the characteristics of both optimized coordinates and localized coordinates. ${ }^{29,41,42}$ Additionally, the Flexible Adaptation of Local COordinates of Nuclei (FALCON) coordinates, ${ }^{43}$ and the local monomer (Lmon) model ${ }^{44-47}$ have also been shown to exhibit considerably less delocalization, compared to the NCs. Further interesting discussions on this topic have recently been given in the literature. ${ }^{48-51}$

Although the simplicity of the rectilinear coordinates is attractive, they inherently have difficulties in describing non-linear vibrational motions of the nuclei and as a result can become overly coupled. It is for this reason that it can be advantageous to introduce a set of generalized coordinates for describing the internal motion of molecular systems, in which both angles and distances feature naturally. ${ }^{52}$ These curvilinear coordinates are of an inherently more localized character than the rectilinear NCs and can therefore generally be 
expected to be less coupled. ${ }^{3}$ The nuclear KEO, however, takes on a far more complicated form, when expressed in curvilinear coordinates, and specialized numerical approaches, as well as analytical approaches have been developed to overcome this problem. The numerical approach, which is based on transforming the Laplace operator by using differential calculus, has successfully been developed and used in many applications. ${ }^{53 ?-65}$ The intent of the analytical approach, on the other hand, is to express the nuclear KEO in a special set of coordinates, e.g. Jacobi, ${ }^{66-69}$ Radau, ${ }^{70-72}$ valence, ${ }^{73}$ hyperspherical ${ }^{74-79}$ or polyspherical,,${ }^{1,80-91}$ in order to arrive at an analytical expression for the nuclear KEO. It is of particular note that parametrizing internal coordinate vectors with polyspherical coordinates (PsCs) ensures that the resulting nuclear KEO will be in the computationally advantageous sum of products form. ${ }^{1}$ Examples of the $\mathrm{PsC}$ parametrization for the formaldehyde and hydrogen peroxide molecules, as employed in this work, are illustrated in fig. 1.

We rely on the analytical approach in this work, through the use of the TANA program. ${ }^{88,90}$ This program is able to both generate a set of PsCs from an arbitrary vector parametrization of the molecular system and provide the associated nuclear KEO in the sum of products form. The PsCs and nuclear KEO are combined with an adaptive algorithm for PES construction and subsequently used in vibrational coupled cluster (VCC) or vibrational configuration interaction (VCI) calculations, as implemented in the Molecular interactions, dynamics and simulations Chemistry program package (MIDASCPP), ${ }^{92}$ in order to determine anharmonic vibrational wave functions. We will in the following section summarize the theoretical basis for determining the nuclear KEO in curvilinear PsCs and in rectilinear NCs. We thereafter give a brief introduction to PES constructing using both rectilinear and curvilinear coordinates and provide a very brief discussion of the integration into VCC theory. Computational details are subsequently provided, followed by a presentation and discussion of our results, before a summary and outlook is given. 


\section{Vibrational Hamilton Operator}

The time-independent Schrödinger equation can be separated into an electronic and a nuclear part by means of the Born-Oppenheimer approximation. ${ }^{93}$ The nuclear Schrödinger equation contains a Hamilton operator

$$
\hat{H}_{\mathrm{nuc}}=\hat{T}_{\mathrm{nuc}}+V(\mathbf{R}),
$$

which consists of a nuclear kinetic energy operator (KEO) and the potential energy surface (PES) for the molecular system. The precise form of the KEO and PES depends on the choice of coordinates, with which the nuclear geometry is expressed. ${ }^{94}$ The nuclear KEO accounts for all $3 N$ nuclear degrees of freedom, namely translational, rotational and vibrational, where $N$ is the number of atoms. It can, however, be worthwhile to adopt a set of coordinates that separates these nuclear degrees of freedom to as large an extent as possible. This can be achieved by adopting the standard set of rectilinear normal coordinates (NCs) and orthogonal transformed coordinate variants thereof, but can equally well be achieved for generalized curvilinear coordinates. Thus, it is possible to treat the vibrational degrees of freedom exclusively by neglecting the rotational contributions. The nuclear KEO can furthermore be obtained in the computationally advantageous sum of products form by adopting the set of curvilinear polyspherical coordinates $(\mathrm{PsCs}) .{ }^{88,90}$

\section{The Polyspherical Approach}

PsCs are a class of curvilinear coordinates, which have been intensively studied over the last few decades, leading to numerous publications, ${ }^{80-91}$ a review, ${ }^{1}$ and book chapters,,${ }^{95,96}$ where various analytical expressions of the nuclear KEO are derived. The main objective of this section, and the associated appendix, is to present the most essential aspects of the PsCs, as relevant for their integration with VCC theory. Note that the application of PsCs, in this work, is carried out in much the same spirit as other recent applications ${ }^{97,98}$.

The PsCs are used to describe internal molecular motion by parametrization of internal 
position vectors, which are projected onto the body-fixed (BF) frame of reference and denoted $\mathbf{R}_{k}^{\mathrm{BF}}$. The set of internal position vectors are parametrized in terms of standard spherical coordinates $R_{k} \in[0, \infty], \theta_{k}^{\mathrm{BF}} \in[0, \pi]$ and $\phi_{k}^{\mathrm{BF}} \in[0,2 \pi]$, i.e. a distance, a valence angle and a dihedral angle. The BF frame of reference is defined in such a way, as to always have the $z^{\mathrm{BF}}$-axis oriented parallel to the first internal position vector $\mathbf{R}_{1}^{\mathrm{BF}}$, while the $(x z)^{\mathrm{BF}}$ plane with a positive $x^{\mathrm{BF}}$ component, i.e. a half-plane, is oriented to be parallel with respect to the second internal position vector $\mathbf{R}_{2}^{\mathrm{BF}} \cdot{ }^{88,90}$ The set of PsCs consist of $N-1$ vector lengths $R_{k}, N-2$ valence angles $\theta_{k}^{\mathrm{BF}}$ and $N-3$ dihedral angles $\phi_{k}^{\mathrm{BF}} .{ }^{81}$ These $M=3 N-6$ coordinates $\tilde{\mathbf{Q}}$ are the PsCs and describe the internal motion of the molecular system based on the specific internal vector parametrization. An illustration of the $\mathrm{PsC}$ parametrization for the formaldehyde and hydrogen peroxide molecules can be found in fig. 1 and may serve as helpful examples of this particular set of curvilinear internal coordinates.
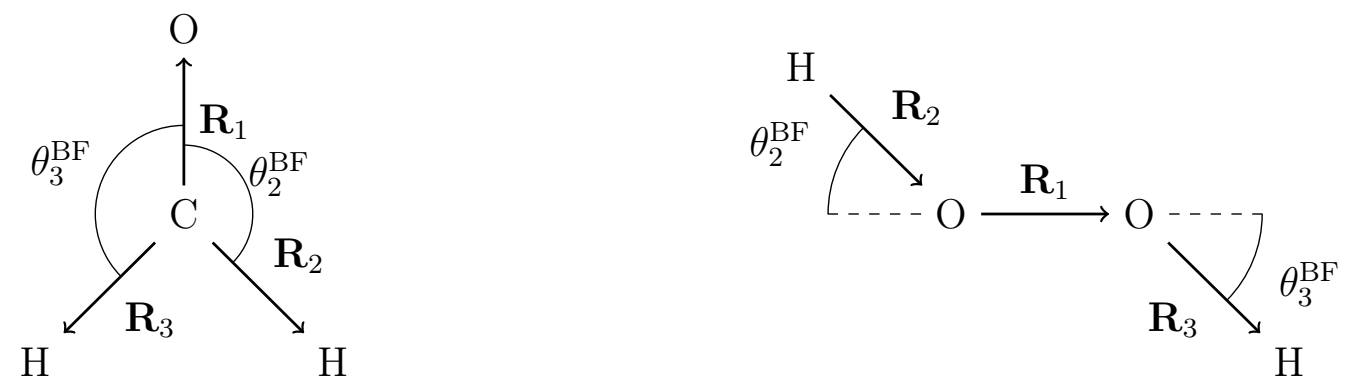

Figure 1: Illustration of the $\mathrm{PsC}$ parametrization for the formaldehyde and hydrogen peroxide molecules, which includes three internal vectors, two valence angles and a single dihedral angle for each molecule. The valence angles $\theta_{2}^{\mathrm{BF}}$ and $\theta_{3}^{\mathrm{BF}}$ describe the angles between internal vectors $\mathbf{R}_{1}^{\mathrm{BF}}$ and $\mathbf{R}_{2}^{\mathrm{BF}}$ or $\mathbf{R}_{3}^{\mathrm{BF}}$, while the dihedral angles $\phi_{3}^{\mathrm{BF}}$ describe the angles between internal vectors $\mathbf{R}_{2}^{\mathrm{BF}}$ and $\mathbf{R}_{3}^{\mathrm{BF}}$ around $\mathbf{R}_{1}^{\mathrm{BF}}$. Note that the dihedral angles are not shown.

The molecular system can be approximated to exclusively be in the rotational ground state, i.e. the total angular momentum operators $\hat{\mathbf{J}}$ are set to zero, if the emphasis is solely on vibrational degrees of freedom. This means that only the deformational part of the nuclear 
KEO needs to be accounted for and leads to an expression for the nuclear KEO of the form

$$
\begin{aligned}
\hat{T}_{\mathrm{nuc}}=- & \frac{\hbar^{2}}{2} \sum_{m, n=1}^{M} \Sigma^{m n}(\tilde{\mathbf{Q}}) \frac{\partial^{2}}{\partial \tilde{Q}_{m} \partial \tilde{Q}_{n}} \\
& -\frac{\hbar^{2}}{2} \sum_{m, n=1}^{M}\left(\Sigma^{m n}(\tilde{\mathbf{Q}})\left(\frac{\partial}{\partial \tilde{Q}_{m}} \ln (\tilde{J}(\tilde{\mathbf{Q}}))+\frac{\partial}{\partial \tilde{Q}_{m}} \Sigma^{m n}(\tilde{\mathbf{Q}})\right) \frac{\partial}{\partial \tilde{Q}_{n}},\right.
\end{aligned}
$$

where $\Sigma^{m n}(\tilde{\mathbf{Q}})$ is a matrix that can be identified from the block matrix form of the contravariant metric matrix. ${ }^{77}$ See the first part of the appendix and references therein for the overall steps in the derivation of this expression of the nuclear KEO and further discussion of the $\Sigma^{m n}(\tilde{\mathbf{Q}})$ matrix.

Additionally, it should be mentioned that it is possible for singularities to occur, in the analytical expression of the nuclear KEO, for specific nuclear configurations, where the valence angle $\theta_{k}^{\mathrm{BF}}=0 \vee \pi$. A solution to this problem, can be to introduce local intermediate $\mathrm{BF}$ frames of reference for a selected subsystem of the full molecular system and singularities can thereby be numerically avoided. ${ }^{81}$ The local intermediate BF frames of reference are associated with a set of vectors, which are a subset of the total $N-1$ internal vectors and two of these subsystem vectors will be used to define the intermediate BF frame of reference. Thus, singularities can be numerically avoided for particular choices of local intermediate $\mathrm{BF}$ frames of reference, at the expense of defining the nuclear KEO recursively, in terms of subsystem nuclear KEOs. ${ }^{1,88,90}$

The TANA program ${ }^{88,90}$ incorporates all the aspects that comprises the polyspherical method, i.e. everything from the initial definition of internal vectors, $\mathrm{PsC}$ parametrization, setting up subsystems and local BF frames of reference, to finally determining the nuclear KEO. An analytical expression, in the sum of products form, is thereby determined for the nuclear KEO in PsCs, which can be provided in a number of different formats, including a MidasCpp ${ }^{92}$ compliant one. This can be achieved for the use of both orthogonal and non-orthogonal internal vectors in the polyspherical approach. The TANA program thus 
facilitates an automatic derivation of an exact nuclear KEO, including all deformational, Coriolis coupling and rotational contributions to the kinetic energy.

\section{The Kinetic Energy Operator for Rectilinear Coordinates}

The internal vibrational motion of rigid or semi-rigid molecules can be adequately described by using rectilinear coordinates, instead of the curvilinear ones, provided that the vibrational modes are of sufficiently small amplitudes, thus lowering the complexity of constructing the nuclear KEO. ${ }^{2,3,96}$

An exact nuclear Hamilton operator based on the rectilinear NCs was derived for nonlinear molecules by Watson, ${ }^{99}$ which we will be using in the form that it takes for a nonrotating molecule, in the same spirit as that applied in the context of eq. (2). This form of the Watson operator is furthermore applicable to any set of coordinates that can be obtained by orthogonal transformation of the NCs, such as the hybrid optimized and localized coordinates (HOLCs). ${ }^{41}$ Thus, we aim to compare the results, as obtained with the use of exact nuclear KEOs for non-rotating molecules in both rectilinear and curvilinear coordinates, in this work.

\section{Potential Energy Surface Construction}

The $M$-dimensional PES can be approximated through the $n$-mode expansion, ${ }^{4-15}$ where the coupling between vibrational modes is restricted. The complete PES can thereby be approximated by a sequence of PESs

$$
V^{(1)}(\mathbf{Q}), V^{(2)}(\mathbf{Q}), \ldots, V^{(M)}(\mathbf{Q})
$$

These approximate PESs can also be denoted as $1 M, 2 M$ or generally $n M$ PESs for which one, two and up to $M$ modes are allowed to be coupled simultaneously, i.e. going from a description given in terms of uncoupled anharmonic oscillators to an exact one. An approx- 
imate PES can be given as

$$
V^{(n)}(\mathbf{Q})=\sum_{\mathbf{m}_{k} \in \operatorname{MCR}[V(\mathbf{Q})]} \bar{V}^{\mathbf{m}_{k}}\left(\mathbf{Q}^{\mathbf{m}_{k}}\right),
$$

where the $k$-dimensional vector $\mathbf{m}_{k}=\left(m_{1}, m_{2}, \ldots, m_{k}\right)$ denotes a mode combination (MC) and contains a set of $k$ modes. ${ }^{100}$ The associated $k$-dimensional vector of the vibrational coordinates $\mathbf{Q}^{\mathbf{m}_{k}}=\left(Q_{m_{1}}, Q_{m_{2}}, \ldots, Q_{m_{k}}\right)$ includes the coordinates of those modes that are active in the particular $\mathrm{MC} \mathbf{m}_{k}$. Note that $\mathbf{Q}$ in this context can denote either a set of rectilinear or curvilinear modes.

The set of MCs that are included in the $n$-mode expansion of the PES are said to constitute a mode combination range (MCR), which determines the level of computational cost for constructing a PES and the accuracy that can be expected of it. ${ }^{15}$ Thus, invoking the $n$-mode expansion restricts the MCR to those index vectors that have, at most, $n$ elements, i.e. all of the included MCs $\mathbf{m}_{k}$ are to comply with $k \leq n$. The general equations and underlying logic of incremental expansions, in this context, have been given several times and the interested reader is referred to refs. 100 and 101.

\section{The Sum of Products Representation}

The PES can be recast, as an analytical expression in the sum of products form, by fitting a direct product of one-mode operators to a grid of single points for each term in eq. (4). The resulting sum of products form for the approximate PESs can thereby be given as

$$
V^{(n)}(\mathbf{Q})=\sum_{\mathbf{m}_{k} \in \operatorname{MCR}[V(\mathbf{Q})]} \sum_{\mathbf{o}_{k}^{\mathbf{m}_{k}}} c_{\mathbf{o}_{k}^{\mathbf{m}_{k}}}^{\mathbf{m}_{k}} \prod_{m \in \mathbf{m}_{k}} \hat{h}_{o^{m}}^{m}
$$

where the individual one-mode operators $\hat{h}_{o^{m}}^{m}$ are indexed by the $o^{m}$ indices for each mode

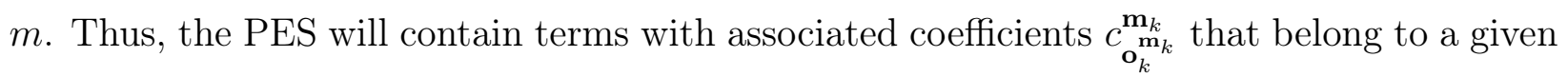
mode $k$ in $\mathbf{m}_{k}$, where the particular operators are given by the $k$-dimensional index vector 
$\mathbf{o}_{k} \mathbf{m}_{k}$. The one-mode operators, which are used to represent the PES, can generally be any type of functions that can provide an adequate representation of the associated bar-potentials and are collectively denoted as the fit-basis. ${ }^{42}$

\section{The Adaptive Density-Guided Approach}

The adaptive density-guided approach (ADGA) ${ }^{23,42,102-105}$ is an iterative black-box procedure for generating a grid of single points, without specialized prior knowledge of a molecular system. The ADGA serves three different purposes to that end; i) generate Cartesian displacement coordinates, ii) establish appropriate grid dimensions, and iii) control grid granularity. The two latter points are achieved via intermediate vibrational self-consistent field $(\mathrm{VSCF})^{7,19-23}$ calculations, which are used to construct the average vibrational density

$$
\rho_{\text {ave }}^{m}\left(Q_{m}\right)=\frac{1}{N_{\text {modals }}^{m}} \sum_{s^{m}=1}^{N_{\text {modals }}^{m}}\left|\varphi_{s^{m}}^{m}\left(Q_{m}\right)\right|^{2}=\frac{1}{N_{\text {modals }}^{m}} \sum_{s^{m}=1}^{N_{\text {modals }}^{m}} \rho_{s^{m}}^{m}\left(Q_{m}\right) .
$$

The orthonormal one-mode wave functions $\varphi_{s^{m}}^{m}\left(Q_{m}\right)$, i.e. functions of only one vibrational coordinate each, are denoted modals in analogy with the orbitals of electronic structure theory. A total of $N_{\text {modals }}^{m}$ modals is said to constitute a basis for the particular mode $m$, i.e. $\left\{\varphi_{s}^{m}\left(Q_{m}\right), s^{m}=1,2, \ldots, N_{\text {modals }}^{m}\right\}$, which are required to be normalized at all times $\left\langle\varphi_{r^{m}}^{m}\left(Q_{m}\right) \mid \varphi_{s^{m}}^{m}\left(Q_{m}\right)\right\rangle=\delta_{r^{m} s^{m}} .{ }^{15}$ The one-mode average vibrational densities $\rho_{\text {ave }}^{m}\left(Q_{m}\right)$ are well-suited to evaluate convergence of one-dimensional grids, while a simple direct product of these are used for evaluating convergence of $n$-dimensional grids

$$
\rho_{\mathrm{ave}}^{\mathbf{m}_{k}}(\mathbf{Q})=\prod_{k^{\prime}=1}^{k} \rho_{\mathrm{ave}}^{m_{k^{\prime}}}\left(Q_{m_{k^{\prime}}}\right)
$$

The one-mode average vibrational densities $\rho_{\text {ave }}^{m}\left(Q_{m}\right)$ are only calculated for the $1 M$ PES in the ADGA and it is thereby easy to construct the direct product of these for $n$-dimensional grids, where $k>1$. 
The relative or absolute change in the energy-like quantities

$$
T_{I_{\ell^{m_{k}}}^{\mathbf{m}_{k}}}^{\mathbf{m}_{k}}=\int_{I_{\ell}^{m_{1}}} \int_{I_{\ell^{m_{2}}}^{m_{2}}} \cdots \int_{I_{\ell^{m_{k}}}^{m_{k}}} \rho_{\text {ave }}^{\mathbf{m}_{k}}(\mathbf{Q}) \bar{V}^{\mathbf{m}_{k}}(\mathbf{Q}) d Q_{m_{1}} d Q_{m_{2}} \cdots d Q_{m_{k}}
$$

are investigated in the ADGA for all internal intervals $I_{\ell^{\mathbf{m}_{k}}}^{\mathbf{m}_{k}}$, as defined by consecutive single points in the grid for a particular MC, when comparing one iteration to the previous one. Furthermore, it is ensured in the ADGA that the vibrational wave function is adequately contained by the grids by taking the sum of integrated average vibrational density for all integration intervals of the individual one-dimensional MC grids.

Following this, there are three convergence criteria for the ADGA, which are controlled by the thresholds $\epsilon_{\text {abs }}, \epsilon_{\text {rel }}$ and $\epsilon_{\rho}$. The ADGA is first considered converged, when the change in average vibrational density and potential energy is smaller than that allowed by either the relative or absolute convergence criteria and a smaller amount of average vibrational density is outside of the grid than that allowed by the density criterion. ${ }^{42}$ Note that the overall form of the evaluated integrals is unchanged, due to the use of Wilson normalization, at the cost of including an extra-potential term. It should, however, be pointed out that the ADGA is only used to construct the PES, while the extra-potential term is always included as part of the nuclear KEO in PsCs.

\section{Cartesian Displacement Coordinates}

Cartesian displacement coordinates for new single points are generated as part of the ADGA and this procedure is different for the use of rectilinear and curvilinear coordinates but the overall workings of the ADGA remains unchanged. Note that the procedure of obtaining the Cartesian displacement coordinates based on rectilinear coordinates have already been described in previous work ${ }^{42}$.

The main difference in obtaining Cartesian displacement coordinates based on curvilinear coordinates is that the natural intervals for which distances, valence angles and dihedral 
angles are defined, i.e. $R_{k} \in[0, \infty], \theta_{k}^{\mathrm{BF}} \in[0, \pi]$ and $\phi_{k}^{\mathrm{BF}} \in[0,2 \pi]$ need to be taken into account explicitly. Displacements along curvilinear vibrational coordinates are therefore carried out by considering each mode in turn that belongs to a given $\mathrm{MC}$ and assign the internal coordinate value as

$$
\tilde{Q}_{m, k^{m_{k}}}^{\mathrm{m}_{k}}= \begin{cases}\tilde{Q}_{m}^{\mathrm{ref}}+\tilde{\mathbf{d}}_{k^{\mathbf{m}_{k}}}^{m}, & \text { if } m \in \mathbf{m}_{k}, \\ \tilde{Q}_{m}^{\mathrm{ref}}, & \text { if } m \notin \mathbf{m}_{k} .\end{cases}
$$

This implies that we are working with the set of polyspherical displacement coordinates $\tilde{\mathbf{d}}$ and not the usual set of PsC $\mathbf{Q}$. Formally, this corresponds to a coordinate transformation and therefore imposes changes on the metric matrix and the nuclear KEO, see Equation (59) of ref. 77. However, since the Jacobian matrix between the two sets of coordinates, i.e. $\tilde{\mathbf{Q}}$ and $\tilde{\mathbf{d}}$, is simply the identity matrix, then the contravariant metric matrix in polyspherical displacement coordinates is expressed by $\mathbf{G}^{\prime}(\tilde{\mathbf{d}})=\mathbf{G}\left(\tilde{\mathbf{Q}}^{\text {ref }}+\tilde{\mathbf{d}}\right)=\mathbf{G}(\tilde{\mathbf{Q}})$. It is thereby possible to transform the nuclear $\mathrm{KEO}$ in $\mathrm{PsC}$ to one in polyspherical displacement coordinates by

simply substituting $\tilde{\mathbf{Q}}$ by $\tilde{\mathbf{Q}}^{\text {ref }}+\tilde{\mathbf{d}}$, without introducing additional or modifying existing terms in the nuclear KEO.

The practical realization of the polyspherical displacement coordinates thereby follows much the same reasoning, as for Cartesian displacement coordinates but with some modifications, as described in the second part of the appendix. After displacing along each curvilinear mode of the given $\mathrm{MC}$, a coordinate transformation from curvilinear to Cartesian ones is carried out via the TANA program. ${ }^{88,90}$

\section{Vibrational Coupled Cluster Theory}

The vibrational coupled cluster (VCC) model ${ }^{22,28,106-108}$ accounts for dynamic correlation between modes, similar to the vibrational configuration interaction (VCI) model, ${ }^{30,109-111}$ but with the possibility of faster convergence towards the full vibrational configuration in- 
teraction (FVCI) limit, when utilizing truncated versions. The VCC wave function ansatz is a non-linear exponential ansatz

$$
|\mathrm{VCC}\rangle=\exp (\hat{T})\left|\Phi_{\mathbf{i}}\right\rangle
$$

where the cluster operator $\hat{T}$ controls excitations out of the reference state

$$
\hat{T}=\hat{T}_{1}+\hat{T}_{2}+\cdots+\hat{T}_{M}=\sum_{\mathbf{m}_{k} \in \operatorname{MCR}[\hat{T}]} \sum_{\mu^{\mathbf{m}_{k}}} t_{\mu^{\mathbf{m}_{k}}} \hat{\tau}_{\mu^{\mathbf{m}_{k}}}
$$

The excitation operator $\hat{\tau}_{\mu^{\mathbf{m}_{k}}}$ enables excitations from occupied to virtual modals and can formally account for all singles, doubles, and up to $M$-tuple excitations, while $t_{\mu^{\mathbf{m}_{k}}}$ is the associated cluster amplitudes.

If no truncation of the cluster operator is introduced, i.e. all $M$-tuple excitations are allowed, then the VCC wave function is identical to the FVCI wave function. This, however, is often not feasible and a hierarchy of progressively more accurate and computationally expensive models are therefore introduced and these are denoted VCC $[n]$ after the truncation level of the MCR. Note that the MCRs, as defined for both PES construction and vibrational structure calculations will formally be similar but that the associated truncation levels need not be the same. However, high-order mode-couplings in the vibrational wave function are typically required if high-order mode-couplings in the Hamilton operator are important.

Moreover, it should be emphasized that even though the present work represents the first case of applying the VCC model in a curvilinear coordinate parametrization, there are no changes introduced into the VCC theory itself. Thus, the VCC model can be employed in exactly the same way as for rectilinear coordinates, due to all significant changes being applied to the Hamilton operator itself and the changes are thus indirect through, for example, new operators and integrals. The non-linear VCC equations are solved using iterative procedures, in order to determine the ground state, as detailed by Madsen et al. ${ }^{112}$. The 
excited states can subsequently be determined via VCC response theory, ${ }^{108,113-116}$ which also remains unchanged under the particular choice of coordinate parametrization, similar to state-specific VCC theory. The analysis of the resulting cluster amplitudes and response vectors will, of course, change due to the $\mathrm{PsC}$ parametrization but this does not introduce changes to the VCC theory itself.

\section{Computational Details}

An interface between the TANA program ${ }^{88,90}$ and the Molecular interactions, dynamics and simulations Chemistry program package (MIDASCPP) ${ }^{92}$ have been implemented and utilized to generated the result presented in the next section. This means that the TANA program have been used for all coordinate transformations, while MiDASCPP have been used for all other purposes, except performing the electronic structure calculations during PES construction. Note that the existing integral evaluation procedures, which are based on the use of B-splines as the primitive basis for representing vibrational wave functions, was extended to cover the specialized cases found in the nuclear KEO in PsCs.

The NCs for formaldehyde were obtained with the TURBOMOLE program ${ }^{117,118}$ by performing geometry optimization and harmonic frequency analysis with the explicitly correlated coupled cluster model with single, double, and scaled perturbative treatment of triple excitations $\left(\mathrm{CCSD}\left(\mathrm{F} 12^{*}\right)\left(\mathrm{T}^{*}\right)\right)^{119}$ using the correlation consistent polarized triple- $\zeta$ basis set, optimized for explicitly correlated methods (cc-pVTZ-F12) ${ }^{120,121}$. The HOLCs were obtained by orthogonal transformation of the NCs in the harmonic potential. The localization penalty weight was set to $w_{P}=1.0 \cdot 10^{-5}$, while the convergence thresholds for coordinate rotation and the numerical Newton minimum search, were both set to $1.0 \cdot 10^{-10}$ a.u. ${ }^{41}$ The PsCs and associated nuclear KEOs for formaldehyde and hydrogen peroxide were obtained by means of the TANA program, ${ }^{88,90}$ using the parametrizations outlined in fig. 1 . The nuclear KEO terms are thereafter handled exclusively through the general functions framework 
in MidASCPP, which was previously introduced in ref. 42 .

The ADGA have been used to obtain up to $6 M$ PESs for formaldehyde and hydrogen peroxide with ADGA convergence thresholds set to $\epsilon_{\text {rel }}=1.0 \cdot 10^{-2}, \epsilon_{\text {abs }}=1.0 \cdot 10^{-6}$ and $\epsilon_{\rho}=$ $1.0 \cdot 10^{-4}$. The relative ADGA convergence criterion were increased by a factor of 20 for the $3 M$ part of the PES, while a factor of 30 was used for the $4 M, 5 M$ and $6 M$ parts of the PES. The initial grid boundaries were set to the turning point for a harmonic oscillator of quantum level $v=2$, in the calculations utilizing $\mathrm{NC}$ and HOLC parametrizations, whereas the initial grid boundaries were set to $0.25 \cdot \sigma^{\tilde{Q}_{m}}$, in the calculations utilizing PsC parametrizations.

A total of 4 modals per mode were used in the VSCF calculations of the average vibrational density for the ADGA, while the primitive basis for each mode consisted of $10010^{\text {th }}$ order B-splines. ${ }^{122}$ The fit-basis consisted of up to $12^{\text {th }}$ order polynomial functions, while the torsional motion for hydrogen peroxide, as characterized by the dihedral angle, was representing by optimized double-well fit-basis functions to the same order. ${ }^{42}$ Note that the reference structure for formaldehyde and hydrogen peroxide, as used in the ADGA, corresponds to the nuclear configuration obtained at the global minimum and the energetically lowest transition state, respectively.

The full Watson operator for a non-rotating molecule, ${ }^{123}$ was used in all vibrational structure calculations based on NC and HOLC parametrizations. The full nuclear KEO in PsCs, was used in all calculations with the PsC parametrization. Fundamental and first overtone excitations were calculated via VCC response theory. ${ }^{108,113-116}$ All VCC calculations have been performed using 8 modals per mode, as determined via VSCF calculations to be the energetically lowest lying, and computed with a primitive basis set of $100 \mathrm{~B}$-splines of order 10 . 


\section{Results}

Results pertaining to the formaldehyde and hydrogen peroxide molecules, see fig. 1, in different coordinate parametrizations will be presented in this section. Results obtained for the formaldehyde molecule will be presented first, while results obtained for the hydrogen peroxide molecule will be presented secondly.

\section{Formaldehyde}

Here follows the results pertaining to the formaldehyde molecule in NC, HOLC and PsC parametrizations. The computational effort in constructing PESs in these coordinate parametrizations are detailed in the first part of this section, while the vibrational excitation energies obtained based on these PESs are presented in the second part of this section.

\section{Constructing the Potential Energy Surface}

The total number of single point calculations (SPCs) required for converging PESs at different MC levels for the three coordinate parametrizations can be found in table 1. It is immediately clear that the PsC parametrization consistently ensures the lowest number of required SPCs at each $\mathrm{MC}$ level. Utilizing the HOLC parametrization results in the highest number of required SPCs for converging the $2 M, 3 M$ and $4 M$ PESs, where this falls to the use of the NC parametrization for the $5 M$ and $6 M$ PESs.

Table 1: The total number of SPCs required for converging PESs at different MC levels based on different coordinate parametrizations for formaldehyde.

\begin{tabular}{ccrccc}
\hline \hline & $2 M$ & $3 M$ & $4 M$ & $5 M$ & $6 M$ \\
\hline NC & 3172 & 11910 & 37648 & 50674 & 50738 \\
HOLC & 3845 & 25479 & 44739 & 45983 & 46047 \\
PsC & 1639 & 6367 & 10805 & 38129 & 38193 \\
\hline \hline
\end{tabular}




\section{Vibrational Excitation Energies}

The fundamental and first overtone excitation energies for formaldehyde were calculated at the FVCI level of theory using fully coupled (6M) PESs based on the three different coordinate parametrizations, see table 2 . These results are quite similar, with a mean absolute deviation $(\mathrm{MAD})$ of less than $1.0 \mathrm{~cm}^{-1}$, when comparing these. The largest deviations are found for the first overtone excitations of highest frequency. These values indicate that the same level of accuracy can be expected for the untruncated treatment, when parametrizing a molecule with different coordinates and constructing the associated PES via the ADGA. The difference in computational effort for constructing the PES is, however, significantly lower when utilizing the $\mathrm{PsC}$ parametrization, as shown in table 1. The remaining deviations in the excitation energies mainly results from the fitting routine and the grid dimension or granularity, as determined in the ADGA for the chosen convergence criteria.

Table 2: Fundamental and first overtone excitation energies for formaldehyde, calculated at the FVCI level of theory based on $6 M$ PESs with different coordinate parametrizations. Mean absolute deviations and maximum absolute deviations are reported for results obtained with the NC parametrization compared to those obtained with HOLC and PsC parametrizations. Excitation energies and deviations are given in $\mathrm{cm}^{-1}$.

\begin{tabular}{clrr}
\hline \hline State & NC & HOLC & \multicolumn{1}{l}{ PSC } \\
\hline$\nu_{1}$ & 1167.81 & 1167.85 & 1168.18 \\
$\nu_{2}$ & 1247.29 & 1247.40 & 1247.40 \\
$\nu_{3}$ & 1499.34 & 1499.46 & 1499.07 \\
$\nu_{4}$ & 1749.72 & 1749.66 & 1749.47 \\
$\nu_{5}$ & 2781.48 & 2781.71 & 2782.71 \\
$\nu_{6}$ & 2842.42 & 2841.85 & 2842.00 \\
$2 \nu_{1}$ & 2328.82 & 2328.64 & 2329.54 \\
$2 \nu_{2}$ & 2491.38 & 2491.35 & 2491.43 \\
$2 \nu_{3}$ & 2998.09 & 2998.19 & 2996.84 \\
$2 \nu_{4}$ & 3480.39 & 3480.25 & 3478.92 \\
$2 \nu_{5}$ & 5464.83 & 5462.69 & 5465.40 \\
$2 \nu_{6}$ & 5651.92 & 5649.01 & 5649.76 \\
\hline $\mathrm{MAD}$ & - & 0.55 & 0.74 \\
$\mathrm{MAX}$ & - & 2.91 & 2.16 \\
\hline \hline
\end{tabular}


The fundamental and first overtone excitation energies were furthermore calculated at different excitation levels in the VCC model, based on PESs of different MC levels for formaldehyde in NC, HOLC and PsC parametrization. All calculated excitation energies for formaldehyde are shown in fig. 2, while the numerical values are provided in tables S-I to S-VII in the supporting information.

It is possible to identify some reoccurring trends for all three sets of coordinates, when inspecting fig. 2, including that the overall expected convergence of the $\operatorname{VCC}[n]$ hierarchy towards the FVCI limit is observed. The VCC[2] model can be seen to actually perform better than the VCC[3] model for the first overtone excitation energies of highest frequency, when employing the NCs, which indicates that state resonances are problematic at this level of theory. The VCC[2] model, on the other hand, clearly performs worse than any of the other $\operatorname{VCC}[n]$ models for the use of HOLCs and a smooth convergence of the $\operatorname{VCC}[n]$ hierarchy can be observed. Turning now to the results obtained with the use of PsC, it can be seen that two of the first overtone excitation energies are clearly not described properly in the VCC[2] model, with errors larger than $100 \mathrm{~cm}^{-1}$, compared to the FVCI results. These excitations involve a coupling between the two valence angles and the $\mathbf{R}_{1}$ coordinate, see fig. 1. This insufficiency probably originates from the excitation level being too small to adequately encompass the associated higher order mode-coupling terms of the nuclear KEO. This particular problem is completely alleviated for the VCC[3] model but the first overtone excitation of highest frequency is, interestingly, relatively poorly described at this level. The $\operatorname{VCC}[n]$ hierarchy, as determined by the excitation level $n$, is generally close to convergence at the $\mathrm{VCC}[4]$ level of theory for all three coordinate parametrizations.

Furthermore, it can be seen in fig. 2 that there is a difference in how the rate of convergence for the excitations energies behaves with respect to MC level of the PES, when taking the effect of the excitation level, as described above, into account. The HOLCs facilitate the best converged results, when comparing the results obtained based on $2 M$ PESs, followed by PsCs and then NCs. This is true when comparing the results obtained based on 3M PESs 
as well, while all results are generally quite close to convergence for the use of $4 M, 5 M$ and $6 M$ PESs.

MADs of the fundamental and first overtone excitation have been determined, in order to further substantiate the analysis of the rate of convergence for the use of the three different coordinate parametrizations, which can be seen in figs. 3 and 4 . It is immediately clear that the first overtone excitations are considerably more demanding to describe accurately than the fundamental excitation energies, as previously discussed. Moreover, it is clearly visible that convergence towards the respective untruncated results is far from achieved, when limited to a $2 M \mathrm{PES}$ or the $\mathrm{VCC}[2]$ model. It can be observed that a calculation using the $\mathrm{VCC}[4]$ model based on a $4 M$ PES is required for obtaining a MAD of less than $1.0 \mathrm{~cm}^{-1}$, when utilizing the NC parametrization. This can already be achieved by using the VCC $[4]$ model based on a $3 M$ PES or the VCC[3] model based on a $4 M$ PES, when considering the HOLC parametrization. Likewise, utilizing the PsC parametrization ensures that this level of convergence is achieved with the use of the $\mathrm{VCC}[4]$ model based on a $3 M$ PES.

Thus, it is demonstrated that the NC parametrization is inferior to both the HOLC and $\mathrm{PsC}$ parametrizations, in terms of ensuring fast convergence of the $\operatorname{VCC}[n]$ hierarchy by way of constructing more suitable PESs. Considering the computational effort required to construct $3 M$ and $4 M$ PESs via the ADGA in HOLC and PsC parametrizations, it can be seen from table 1 that considerably fewer SPCs are needed for the use of PsCs. It is thereby, for formaldehyde, computationally less costly to use the PsC parametrization, which furthermore does not compromise the accuracy of the vibrational structure calculation either.

We expect that this gain can be exploited for larger molecules as well, where truncation in the MC levels of the PES and in the excitation level of vibrational structure calculations are needed. A fast convergence, with regards to both hierarchies of truncation, is seemingly assured for the use of PsCs, especially for fundamental excitation energies. Note that this approach can easily be combined with a rigid or semi-rigid model for the molecular system, 

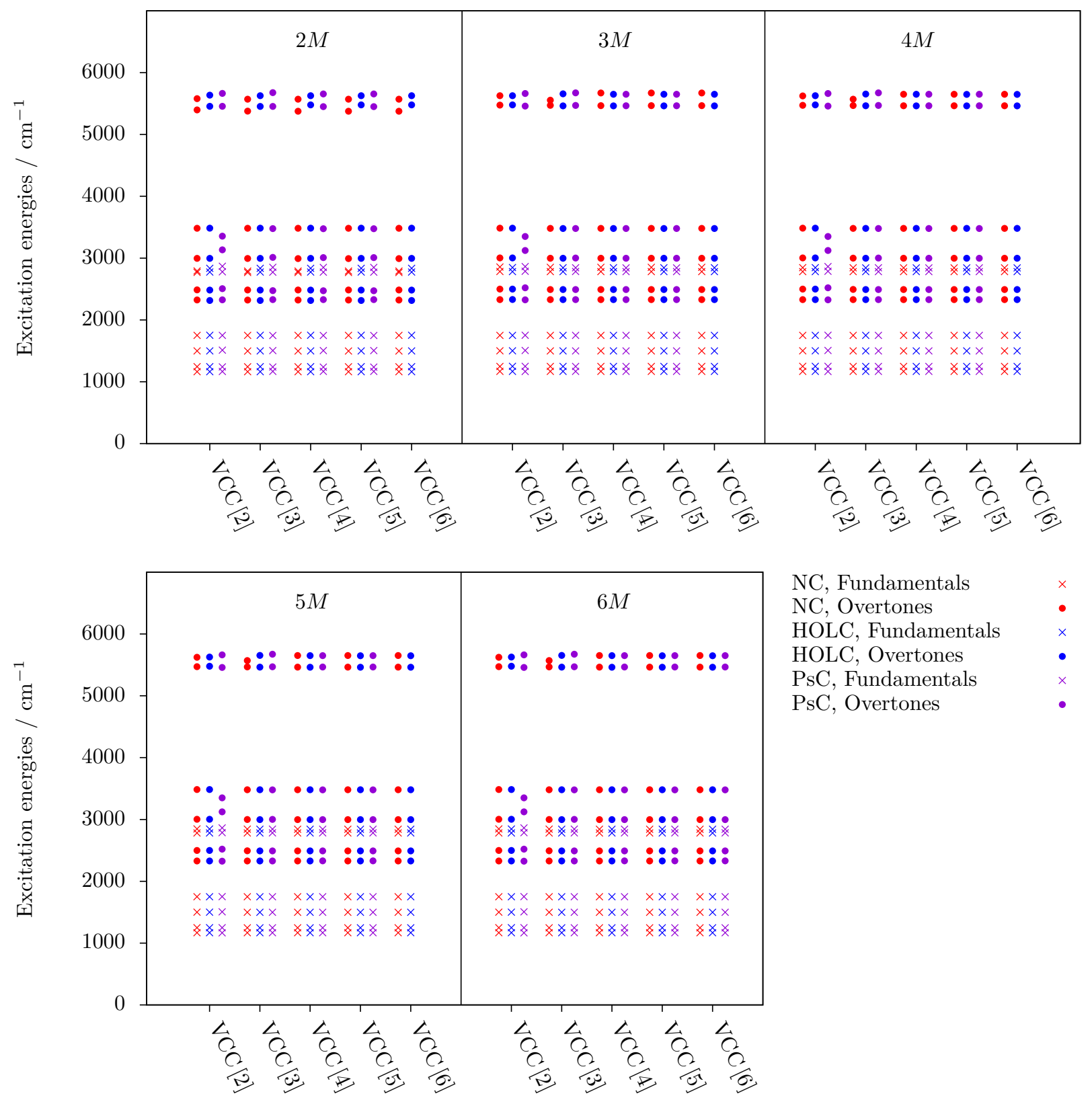

NC, Fundamentals $\quad \times$

NC, Overtones

HOLC, Fundamentals $\times$

HOLC, Overtones •

PsC, Fundamentals $\quad \times$
PsC, Overtones

Figure 2: Fundamental and first overtone excitation energies, calculated with different VCC $[n]$ models based on PESs of different MC levels in different coordinate parametrizations for formaldehyde. 
in which some vibrational coordinates are held inactive or frozen. ${ }^{124}$ This will result in a very efficient $a b$ initio model for computing anharmonic vibrational wave functions.

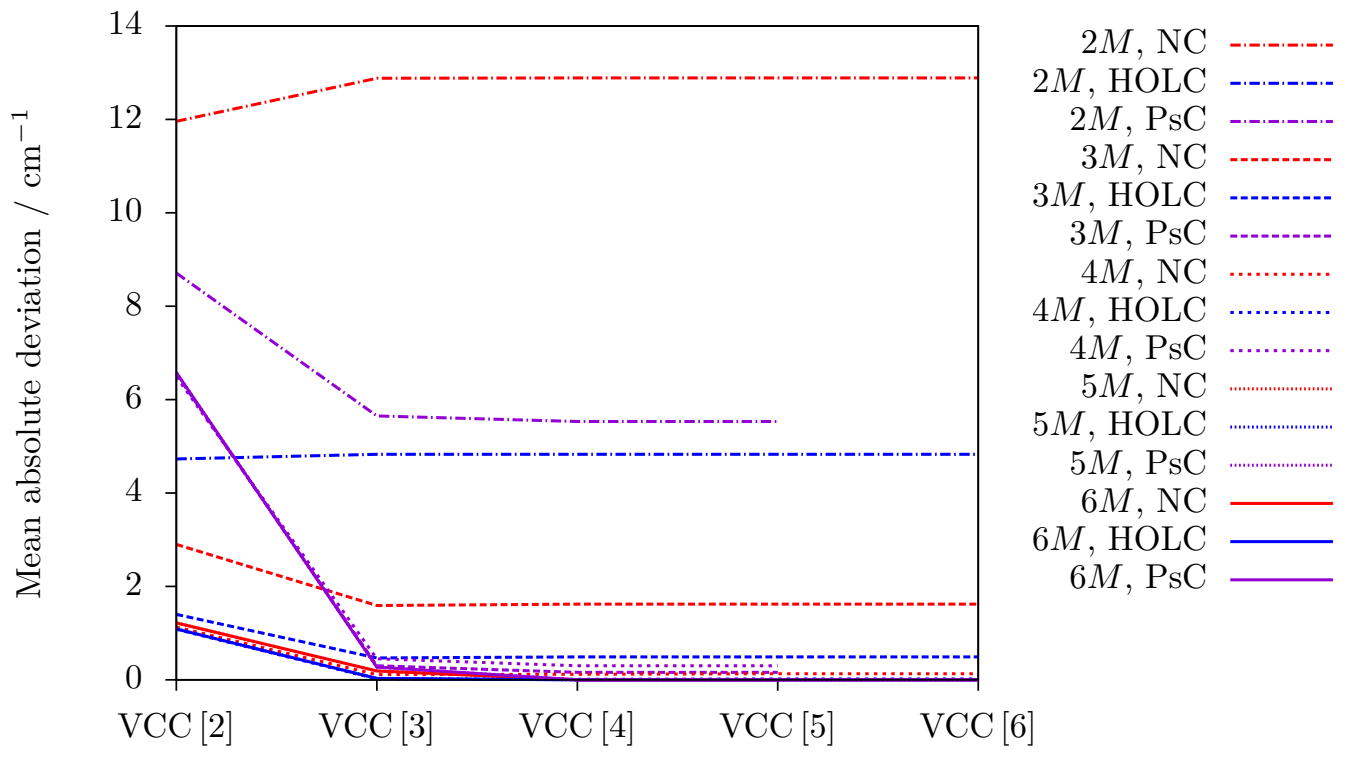

Figure 3: Mean absolute deviations of fundamental excitation energies, calculated with different VCC $[n]$ models based on PESs of different MC levels, compared to calculated FVCI results in the respective coordinate parametrizations for formaldehyde.

\section{Hydrogen Peroxide}

Hydrogen peroxide have attracted much attention over the years, due to its natural abundance and many uses, but also as a molecule for testing computational methods and their ability to treat internal rotations. ${ }^{125}$ The ro-vibrational gas-phase spectrum of hydrogen peroxide have been the subject of many experimental studies, ${ }^{126-131}$ as well as theoretical studies $^{125,132-137}$ and therefore represents a good and non-trivial test of the methods based on PsCs. It should, however, be emphasize that the primitive basis, which is used for representing the vibrational wave function, is constituted by B-splines ${ }^{138,139}$ that are generated from clamped knot sequences and these are, hence, not periodic in nature. ${ }^{140-142}$ The ADGA is, accordingly, restricted to not being able to take full account of the periodicity associated with the valence and dihedral angles. 


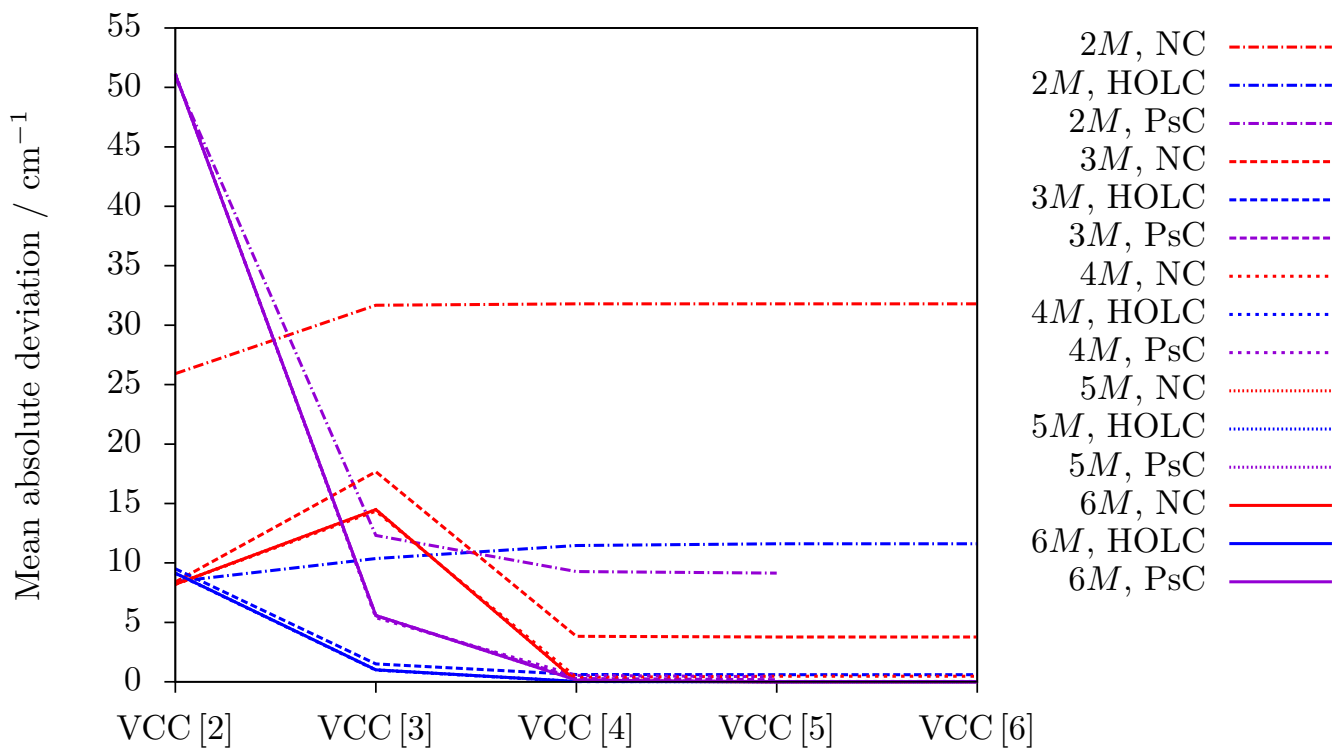

Figure 4: Mean absolute deviations of first overtone excitation energies, calculated with different VCC $[n]$ models based on PESs of different MC levels, compared to calculated FVCI results in the respective coordinate parametrizations for formaldehyde.

We choose to restrict ourselves in treating the hydrogen peroxide molecule in the $\mathrm{PsC}$ parametrization only, see fig. 1, in order to not waste computational efforts. It is, of course, perfectly adequate to treat any molecular system in an arbitrary coordinate parametrization, when considering the limit of using FVCI based on a complete PES. The truncated PESs will, however, be inherently poor for the case of hydrogen peroxide in a rectilinear coordinate parametrization.

\section{Constructing the Potential Energy Surface}

Comparing the number of SPCs required for converging PESs at different MC levels for hydrogen peroxide and formaldehyde in their respective $\mathrm{PsC}$ parametrizations, as seen in tables 1 and 3 , it is clear that the distributions of grid points is somewhat different. The $2 M$, $3 M$ and $4 M$ PESs for hydrogen peroxide require significantly more SPCs to converge in the ADGA, while the $5 M$ and $6 M$ PESs require fewer than that needed for the corresponding PESs for formaldehyde. The former behaviour is ascribed to the more difficult internal motion that is characterized by the dihedral angle, while the latter behaviour is, perhaps, 
due to the use of a more suitable fit-basis for this particular motion. Both observations are very interesting and should be explored further in the context of different computational set-ups and for different molecular systems.

Table 3: The total number of SPCs required for converging PESs at different MC levels based on the PsC parametrization for hydrogen peroxide.

\begin{tabular}{cccccc}
\hline \hline & $2 M$ & $3 M$ & $4 M$ & $5 M$ & $6 M$ \\
\hline $\mathrm{PsC}$ & 4454 & 11962 & 22167 & 35665 & 35729 \\
\hline \hline
\end{tabular}

\section{Vibrational Excitation Energies}

The ground state energy, fundamental and first overtone excitation energies with a frequency below $4000 \mathrm{~cm}^{-1}$ were calculated for hydrogen peroxide, at the FVCI level of theory using a fully coupled (6M) PES based on the PsC parametrization, which can be seen in table 4. Note that we follow the state labelling previously introduced by Małyszek and Koput. ${ }^{125}$ The majority of the calculated excitation energies reproduce the experimentally observed values quite well, as can be ascertained from the MAD of only $4.56 \mathrm{~cm}^{-1}$, but with some notable outliers. These outliers are, in particular, found for the $\nu_{3}$ states, which are associated with the $\mathrm{O}-\mathrm{O}$ stretching motion and exhibits the maximum absolute deviations (MAXs). The states $\nu_{2}$ and $\nu_{6}$, which are associated with the symmetrical and asymmetrical $\mathrm{O}-\mathrm{O}-\mathrm{H}$ bending motions respectively, are quite accurately determined, while the states $\nu_{1}$ and $\nu_{5}$, which are associated with the symmetrical and asymmetrical $\mathrm{O}-\mathrm{H}$ stretching motions, are a little less accurately determined. The $\nu_{4}$ states, which are associated with the torsional motion of hydrogen peroxide are furthermore quite well reproduced and even the tunnelling splitting is correctly accounted for by the VCC [6] model.

The calculated energies can be regarded as being almost converged, in an absolute sense, when taking into account the various small effects, such as relativistic corrections, considered by Małyszek and Koput. ${ }^{125}$ The magnitude and sign of these corrections match the errors of our calculated results very well. This is especially true for the $\nu_{3}$ states, where the 
corrections are most prominent and can be larger than $10 \mathrm{~cm}^{-1}$. This underlines that the vibrational structure of hydrogen peroxide can be correctly represented by the VCC model, when utilizing an adequate PES representation based on a suitable coordinate parametrization. The PsC parametrization is such a parametrization and provides a natural account of the internal motion, with which the ADGA and VCC model can be applied for efficient and accurate calculations.

Table 4: Absolute deviations of calculated and experimentally observed ground state energies, fundamental and first overtone excitation energies with a frequency below $4000 \mathrm{~cm}^{-1}$ for hydrogen peroxide. The calculated values are determined with the VCC [6] model based on a $6 M$ PES in the PsC parametrization. MAD and the maximum absolute deviation are furthermore provided. All numbers are given in $\mathrm{cm}^{-1}$.

\begin{tabular}{|c|c|c|c|c|}
\hline State & Symm. & Calc. & Dev. & Obs. \\
\hline $0^{+}$ & $A_{q}$ & 0.00 & 0.00 & 0.00 \\
\hline $0^{-}$ & $A_{u}$ & 9.98 & 1.42 & $11.40^{127}$ \\
\hline$\nu_{4}^{+}$ & $A_{g}$ & 260.48 & 5.88 & $254.60^{127}$ \\
\hline$\nu_{4}^{-}$ & $A_{u}$ & 370.64 & 0.26 & $370.90^{127}$ \\
\hline $2 \nu_{4}^{+}$ & $A_{g}$ & 570.79 & 1.09 & $569.70^{127}$ \\
\hline $2 \nu_{4}^{-}$ & $A_{u}$ & 776.10 & 0.00 & $776.10^{127}$ \\
\hline$\nu_{3}^{+}$ & $A_{g}$ & 877.67 & 11.77 & $865.90^{129}$ \\
\hline$\nu_{3}^{-}$ & $A_{u}$ & 888.05 & 10.15 & $877.90^{129}$ \\
\hline$\nu_{6}^{+}$ & $B_{u}$ & 1266.39 & 1.79 & $1264.60^{128,130}$ \\
\hline$\nu_{6}^{-}$ & $B_{g}$ & 1288.78 & 3.68 & $1285.10^{128,130}$ \\
\hline$\nu_{2}^{+}$ & $A_{g}$ & 1398.34 & 2.44 & $1395.90^{128,130}$ \\
\hline$\nu_{2}^{-}$ & $A_{u}$ & 1403.92 & 5.62 & $1398.30^{128,130}$ \\
\hline $2 \nu_{6}^{+}$ & $A_{g}$ & 2509.48 & 3.18 & $2506.30^{126}$ \\
\hline $2 \nu_{6}^{-}$ & $A_{u}$ & 2548.14 & 8.84 & $2539.30^{126}$ \\
\hline $2 \nu_{2}^{+}$ & $A_{q}$ & 2758.60 & 7.40 & $2766.00^{126}$ \\
\hline $2 \nu_{2}^{-}$ & $A_{u}$ & 2774.27 & 4.77 & $2769.50^{126}$ \\
\hline$\nu_{1}^{+}$ & $A_{g}$ & 3611.08 & 1.28 & $3609.80^{126}$ \\
\hline$\nu_{5}^{+}$ & $B_{u}$ & 3614.78 & 4.89 & $3610.70^{126}$ \\
\hline$\nu_{1}^{-}$ & $A_{u}$ & 3615.59 & 4.71 & $3618.00^{126}$ \\
\hline$\nu_{5}^{-}$ & $B_{g}$ & 3622.71 & 7.49 & $3618.80^{126}$ \\
\hline \multicolumn{2}{|c|}{ MAD } & - & 4.56 & - \\
\hline \multicolumn{2}{|c|}{ MAX } & - & 11.77 & - \\
\hline
\end{tabular}

The fundamental and first overtone excitation energies with a frequency below $4000 \mathrm{~cm}^{-1}$, 
were calculated at different excitation levels in the VCC model, based on PESs of different $\mathrm{MC}$ levels for hydrogen peroxide in the $\mathrm{PsC}$ parametrization. All calculated excitation energies for hydrogen peroxide are shown in fig. 5, while the numerical values are provided in tables S-VIII to S-XII of the supporting information.

The same pattern, as previous noted in connection with formaldehyde in the $\mathrm{PsC}$ parametrization, can be seen for hydrogen peroxide in the $\mathrm{PsC}$ parametrization, when inspecting fig. 5. It can be seen that using a $2 M$ PES does not allow for obtaining reasonably converged excitation energies, which is also the case for the use of the $\operatorname{VCC}[2]$ model. Using the $\operatorname{VCC}[4]$ model, on the other hand, ensures very close to converged values for the $4 M, 5 M$ and $6 M$ PESs. It is furthermore interesting to note that the ground state torsional splitting, i.e. the tunnelling splitting, is consistently well described by the VCC model and it is, in fact, already converged to below $1.0 \mathrm{~cm}^{-1}$, when applying the $\mathrm{VCC}[2]$ model based on a $2 M$ PES. This observation can be attributed to the use of a truly adequate set of vibrational coordinates and the use of an appropriate analytical representation.

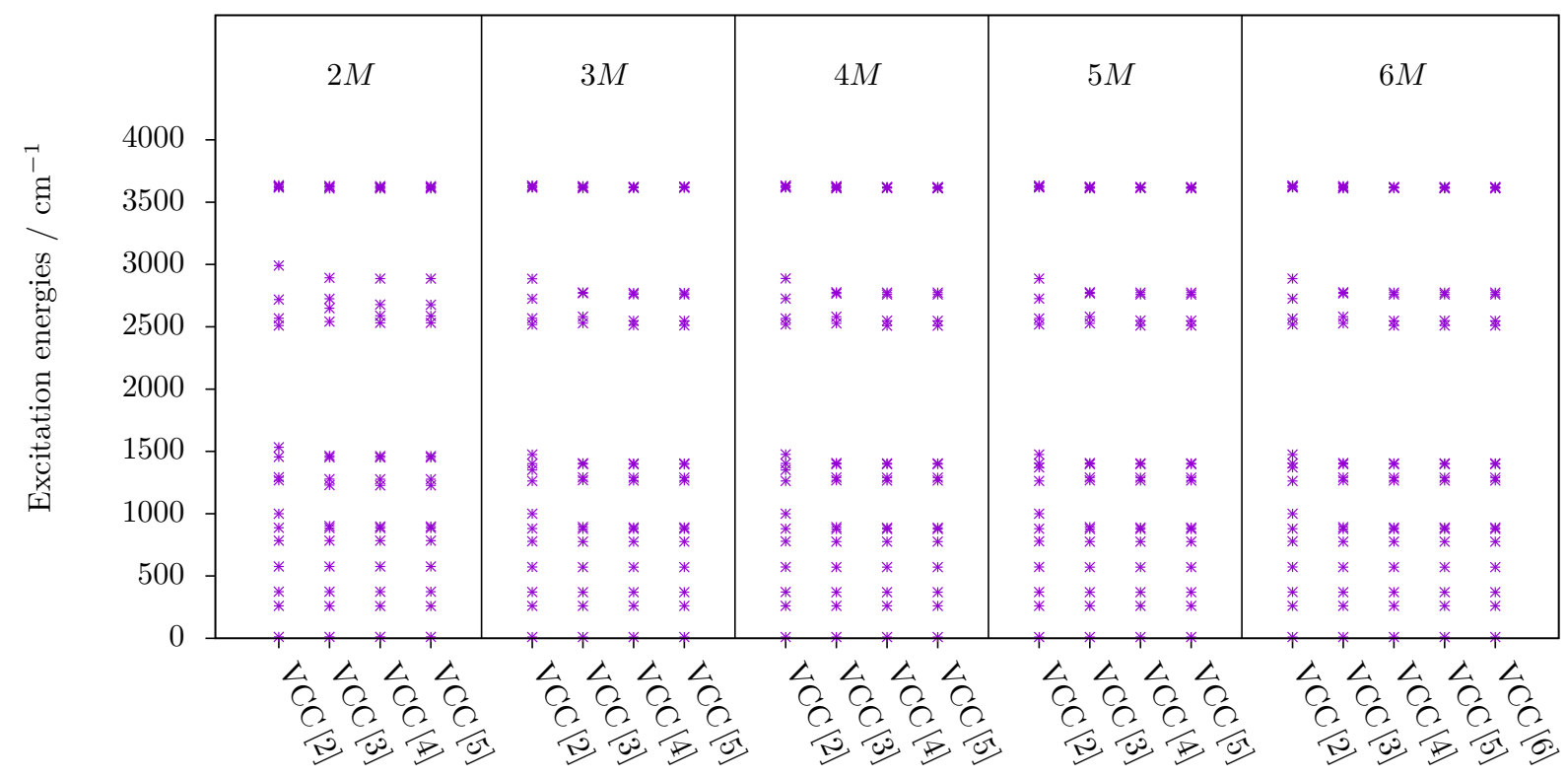

Figure 5: Ground state energies, fundamental and first overtone excitation energies with a frequency below $4000 \mathrm{~cm}^{-1}$, calculated with different $\operatorname{VCC}[n]$ models based on PESs of different $\mathrm{MC}$ levels for hydrogen peroxide in the PsC parametrization. 
MADs of the calculated energies, as seen in fig. 5, was compared to the energies obtained via FVCI based on a $6 M \mathrm{PES}$ and are given in fig. 6 for hydrogen peroxide. This allows for determining if the rate of convergence for the set of calculations performed for hydrogen peroxide in the $\mathrm{PsC}$ parametrization is similar to that observed for formaldehyde. It is immediately clear, from inspecting fig. 6 that calculations on the hydrogen peroxide molecule is much more demanding than calculations on the formaldehyde molecule. It can be seen that, although the convergence is smooth, the results are first converged to below $1.0 \mathrm{~cm}^{-1}$, when applying the VCC[4] model based on a $4 M$ PES.

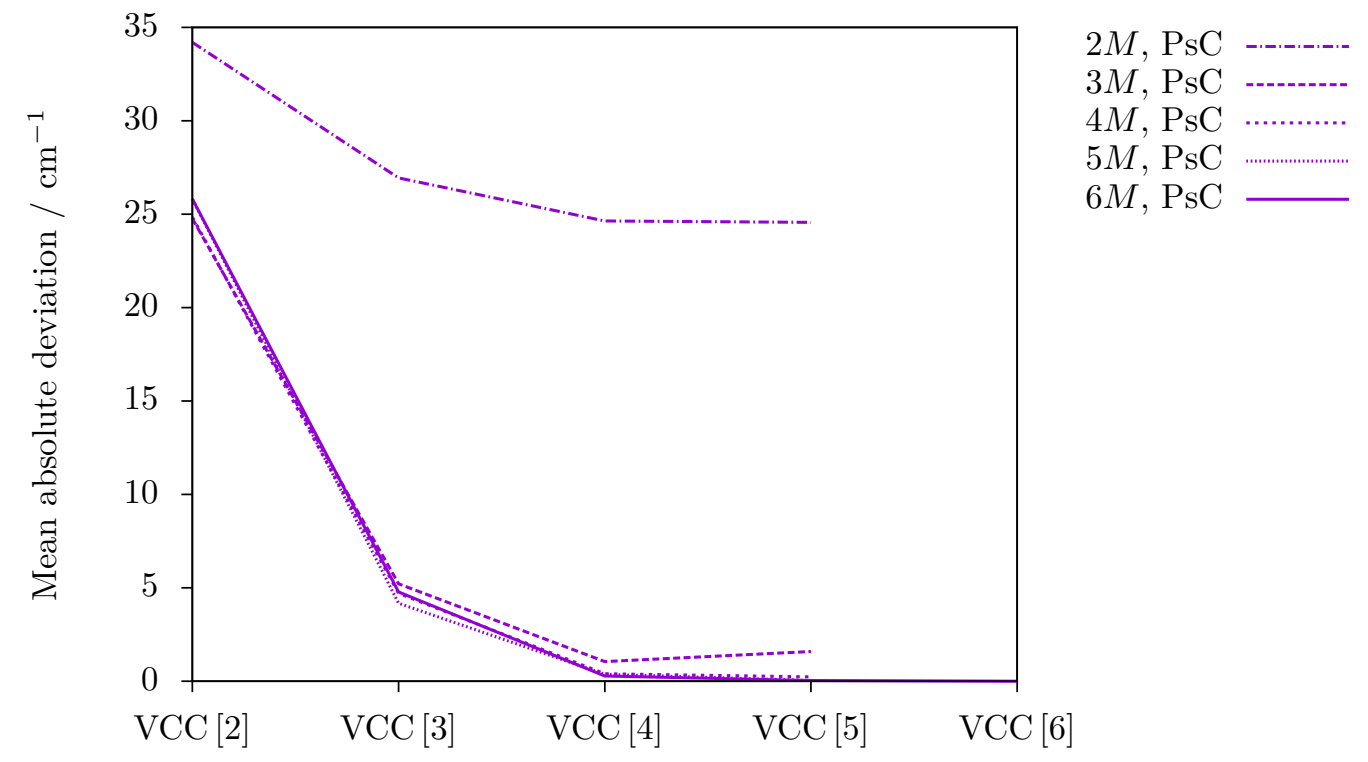

Figure 6: Mean absolute deviations of ground state energies, fundamental and first overtone excitation energies with a frequency below $4000 \mathrm{~cm}^{-1}$, calculated with different $\mathrm{VCC}[n]$ models based on PESs of different MC levels for hydrogen peroxide in the PsC parametrization. These are compared to the energies obtained from a FVCI calculation based on the $6 M$ PES for hydrogen peroxide in the PsC parametrization.

The present computations for hydrogen peroxide, though lacking some electronic corrections, show that this non-trivial case can be handled correctly with the ADGA and VCC models in the PsC parametrization. Furthermore, these results lend themselves to the credibility of the interface between MIDASCPP and the TANA program, which is shown to produce correct results, in an efficient manner. 


\section{Summary and Outlook}

We have presented results and the computational set-up, pertaining to the use of curvilinear PsC in the context of VCC theory and the ADGA to PES construction. This is facilitated through an interface between the MIDASCPP and the TANA program, which allows for performing displacements along curvilinear PsCs during PES construction. The MIDASCPP has been adapted, in order to handle the analytical expression of the nuclear KEO in PsC, which is generated via the TANA program in the computationally advantageous sum of products form.

We have shown that the use of PsCs facilitates a decrease in the number of SPCs required for converging a PES with the ADGA, due to a decrease in the level of coupling between vibrational modes, compared to the use of rectilinear coordinates, namely NCs and HOLCs. Furthermore, we have shown that the $\mathrm{PsC}$ parametrization facilitates an accelerated rate of convergence towards the FVCI limit in vibrational structure calculations, especially when compared to the use of NCs. This means that fairly converged results can be obtained with the VCC model at low orders of approximation, when utilizing the PsCs. It has additionally been shown that the ADGA, based on a PsC parametrization, is able to treat highly nontrivial cases, such as the hydrogen peroxide molecule. This could be achieved, so as to accurately determine the ground state splitting, even at low levels of approximation.

It could be interesting to repeat the calculations on hydrogen peroxide, while using more sophisticated electronic structure methods, in order to ascertain the accuracy of our approach to an even larger degree. Performing such a series of calculations would additionally call for the use of periodic B-splines, which are to be used as primitive basis for the vibrational wave function, in order to adequately represent the internal motion of hydrogen peroxide. This is to be contrasted with the performance of the non-periodic B-splines, as employed in the present work.

Moreover, it would be interesting to apply the PsCs in calculations of medium to large sized molecules in full dimensionality, either on their own or in comparison with different 
rectilinear sets of coordinates. A comparison to the performance of FALCON coordinates would, in this regard, be of special interest, as this set of rectilinear coordinates are known for a large degree of locality. ${ }^{43,143}$ It has already been demonstrated that the TANA program is able to handle molecules as large as benzopyran ${ }^{88}$ and even larger molecules can be considered. The MidAsCPP have been demonstrated to be able to handle molecules with up to hundredths of modes in both PES construction, using the ADGA, ${ }^{23}$ and in VCC calculations of the anharmonic vibrational wave function. ${ }^{144}$ So far the largest of such molecular systems have only been treated at the level of two-mode couplings, while using low-accuracy electronic structure methods. Recent progress indicates that in the future similar high-accuracy computations can be made feasible from developments in integration of machine learning ${ }^{105}$ and incremental expansion methods ${ }^{101,145}$ for PES construction and tensor decomposition methods for anharmonic wave function calculations. ${ }^{112}$ These prospects also apply to the use of PsCs and potentially extends the impact of these.

Formally the increase in computational cost with increasing system size will be the same for PES construction and VCC computations, apart from the kinetic energy treatment, for both NCs and PsCs. The concrete polynomial scalings have been detailed in previous work for both PES construction ${ }^{43}$ and VCC computations. ${ }^{108,144}$ However, here the faster convergence for the truncations of both PES and vibrational structure calculations when utilizing the PsC parametrization, as observed in this work, is key. This, together with the possibility of exploiting the inherent locality of the PsCs and their ability to treat large amplitude motions, indicates that a lower computational cost may be achieved for the same accuracy. Consequently, the PsCs become attractive for many uses, including the treatment of biomolecules, such as amino-acids and peptides, where torsional motions are important. It would also be interesting, in the context of approaching larger sized molecular systems, to explore the frozen mode approximation and the ability to define groups of subsystems for the generation of $\mathrm{PsCs}$ and the nuclear KEO. These possibilities already exist in the TANA program and would be logical additions to the now-established interface to MiDASCPP. 
There is, however, one important reservation to the above considerations, namely that the nuclear KEO was always exact in this work, while the truncation of the PES and the VCC model was explored. It would be an interesting topic of future research to study a sequence of systematic approximations to the nuclear KEO with respect to the level of mode-coupling. The exact analytical nuclear KEO can be handled in a relatively straightforward manner for smaller molecular systems, like those treated in the present study, but this can become problematic for larger systems, due to the presence of terms with high levels of mode-coupling. Eventually this could lead to the computational scaling of treating the kinetic energy parts being more costly than treating the PES part, when the former has a substantially higher coupling level. However, a key result in this study is that only a modest level of modecoupling is needed for the PES and for the VCC model of the anharmonic vibrational wave function, in order to obtain well-converged results. It seems plausible, based on these results that the nuclear KEO can be approximated to similar levels of mode-coupling. Indeed, if such limited mode-coupled nuclear KEO can be automatically constructed, while ensuring the Hermiticity of the Hamilton operator, it would be possible to treat large molecular systems through the combination of the ADGA and the VCC model with PsCs.

\section{Supporting Information Available}

A complete set of data for the results presented as part of this work is provided in the supporting information, as indicated throughout the Results section. The constructed PESs are furthermore provided in their fitted form, along with the analytical form of the nuclear KEO, which can be used to evaluate the molecular energy by providing the internal coordinates to this function. 


\section{Acknowledgement}

O. C. acknowledges support from the Danish e-infrastructure Cooperation (DeiC), and the Danish Council for Independent Research through a Sapere Aude III Grant (No. DFF-400200015). E. L. K. acknowledges support from the molecules in motion (MOLIM) network for a short term scientific mission.

\section{Appendix}

Here follows an account of the steps in the derivation of the nuclear KEO in PsCs, as used in generating the presented results. Considerations regarding the practice of changing normalization and the introduction of extra-potential terms in the nuclear KEO are furthermore provided. This is followed by a small technical note on the generation of polyspherical displacement coordinates in the context of the ADGA.

\section{Deriving the Nuclear Kinetic Energy Operator in Polyspherical Co- ordinates}

The first step of the polyspherical approach is to introduce the $N$ nuclear position vectors in the laboratory-fixed (LF) frame of reference, which points from the origin of the LF frame of reference towards each nucleus $\mathbf{R}_{1}^{\mathrm{LF}}, \mathbf{R}_{2}^{\mathrm{LF}}, \ldots, \mathbf{R}_{N}^{\mathrm{LF}}$. The second step is to define a set of internal position vectors $\mathbf{R}_{1}^{\mathrm{SF}}, \mathbf{R}_{2}^{\mathrm{SF}}, \ldots, \mathbf{R}_{N-1}^{\mathrm{SF}}$, which describe the nuclear configuration in terms of distances in a space-fixed (SF) frame of reference. The last vector of this second vector parametrization points to the molecular center of mass $(\mathrm{CoM}) \mathbf{R}_{N}^{\mathrm{SF}}=\mathbf{R}_{\mathrm{CoM}}^{\mathrm{SF}}$ but this can separated out by enforcing the CoM constraints, ${ }^{146,147}$ thus separating out translational degrees of freedom from rotational and vibrational ones. These two sets of vectors can be 
related through a linear transformation

$$
\mathbf{R}_{k}^{\mathrm{SF}}=\sum_{\kappa=1}^{N} A_{k \kappa} \mathbf{R}_{\kappa}^{\mathrm{LF}},
$$

where $\mathbf{A}$ is the associated $(N-1) \times N$ transformation matrix. ${ }^{81}$ This coordinate transformation entails a transformation of the momentum operators

$$
\hat{\mathbf{p}}_{\kappa}^{\mathrm{LF}}=\sum_{\kappa=1}^{N} A_{\kappa k} \hat{\mathbf{p}}_{k}^{\mathrm{SF}}
$$

which indicates that the transformation matrix is identical to the Jacobian matrix for coordinate transformation from the SF internal vectors to the LF position vectors. It is thereafter possible to construct the constant mass-dependent symmetric $(N-1) \times(N-1)$ matrix

$$
\tilde{M}^{k l}=\sum_{\kappa=1}^{N} A_{k \kappa} m_{\kappa}^{-1} A_{\kappa l}
$$

from the nuclear masses $m_{\kappa}$, the transformation matrix $\mathbf{A}$ and associated transpose $\mathbf{A}^{\mathrm{T}}$. An expression for the nuclear KEO in the internal vector parametrization, which contains the deformational, Coriolis coupling and rotational contributions to the kinetic energy, can then be given in terms of

$$
\hat{T}_{\mathrm{nuc}}=\frac{1}{2} \sum_{k, l=1}^{N-1}\left(\hat{\mathbf{p}}_{k}^{\mathrm{SF}}\right)^{\dagger} \tilde{M}^{k l} \hat{\mathbf{p}}_{l}^{\mathrm{SF}}
$$

The matrix $\tilde{\mathbf{M}}=\mathbf{A} \mathbf{m}^{-1} \mathbf{A}^{\mathrm{T}}$ has a key role in setting up the nuclear KEO by assuming the role of the covariant metric matrix for the nuclear KEO in the internal vector parametrization. ${ }^{52}$ If another set of internal coordinate vectors should be desired, then it is a simple matter of recalculating the Jacobian matrix, i.e. the transformation matrix A. Furthermore, if the set of internal vectors is orthogonal, then $\tilde{\mathbf{M}}$ becomes diagonal and the expression for the nuclear KEO greatly simplifies, see Gatti and $\operatorname{Iung}^{1}$ for explicit examples.

The third step of the polyspherical approach is to introduce a BF frame of reference, in 
order to achieve a separation of overall rotation from internal deformation, i.e. vibrational degrees of freedom. The momentum operators conjugated to the SF internal coordinate vectors are, to that end, projected unto the BF frame of reference. Furthermore, this entails that the momentum operators conjugated to the internal coordinate vectors of the BF frame of reference can be expressed in terms of radial and angular contributions

$$
\begin{gathered}
\hat{\mathbf{p}}_{k}^{\mathrm{BF}}=\hat{p}_{R_{k}} \mathbf{e}_{k}^{\mathrm{BF}}-\frac{\mathbf{e}_{k}^{\mathrm{BF}} \times \hat{\mathbf{L}}_{k}^{\mathrm{BF}}}{R_{k}}, \\
\left(\hat{\mathbf{p}}_{k}^{\mathrm{BF}}\right)^{\dagger}=\hat{p}_{R_{k}}^{\dagger} \mathbf{e}_{k}^{\mathrm{BF}}+\frac{\left(\hat{\mathbf{L}}_{k}^{\mathrm{BF}}\right)^{\dagger} \times \mathbf{e}_{k}^{\mathrm{BF}}}{R_{k}} .
\end{gathered}
$$

The unit vectors along each $\mathbf{R}_{k}^{\mathrm{BF}}$ are denoted $\mathbf{e}_{k}^{\mathrm{BF}}=\mathbf{R}_{k}^{\mathrm{BF}} / R_{k}$ and the projection of vector $\mathbf{R}_{k}^{\mathrm{BF}}$ unto the BF frame of reference, can thereby be given in terms of $\mathbf{R}_{k}^{\mathrm{BF}}=R_{k} \mathbf{e}_{k}^{\mathrm{BF}}$, where $R_{k}$ is the length of the vector $\mathbf{R}_{k}^{\mathrm{BF}}$. The radial momentum operators are given in the usual sense, i.e. $\hat{p}_{R_{k}}=-i \hbar \partial / \partial R_{k}^{\mathrm{BF}}$, while the angular momentum operators $\hat{\mathbf{L}}_{k}$ are quasi-momentum operators. ${ }^{52}$

The fourth step of the polyspherical approach is to parametrize the internal coordinate vectors $\mathbf{R}_{k}^{\mathrm{BF}}$, in terms of PsCs. This means that each of the $N-1$ internal vectors are characterized by spherical coordinates $R_{k} \in[0, \infty], \theta_{k}^{\mathrm{BF}} \in[0, \pi]$ and $\phi_{k}^{\mathrm{BF}} \in[0,2 \pi]$, i.e. a distance, a valence angle and a dihedral angle. The BF frame of reference relies on the Euler angles, in order to describe the orientation relative to the SF frame of reference but is defined in a particular way in the $\mathrm{PsC}$ parametrization. Thus, the $z^{\mathrm{BF}}$-axis of the $\mathrm{BF}$ frame of reference is always oriented parallel to the first internal position vector $\mathbf{R}_{1}^{\mathrm{BF}}$, while the $(x z)^{\mathrm{BF}}$-plane with a positive $x^{\mathrm{BF}}$ component, i.e. a half-plane, is oriented to be parallel with respect to the second internal position vector $\mathbf{R}_{2}^{\mathrm{BF}}$. The unit vectors along the $N-1$ 
internal vectors that define the PsCs in the $\mathrm{BF}$ frame of reference are thereby given as

$$
\mathbf{e}_{1}^{\mathrm{BF}}=\left(\begin{array}{l}
0 \\
0 \\
1
\end{array}\right), \quad \mathbf{e}_{2}^{\mathrm{BF}}=\left(\begin{array}{c}
\sin \left(\theta_{2}^{\mathrm{BF}}\right) \\
0 \\
\cos \left(\theta_{2}^{\mathrm{BF}}\right)
\end{array}\right), \quad \mathbf{e}_{k>2}^{\mathrm{BF}}=\left(\begin{array}{c}
\sin \left(\theta_{k}^{\mathrm{BF}}\right) \cos \left(\phi_{k}^{\mathrm{BF}}\right) \\
\sin \left(\theta_{k}^{\mathrm{BF}}\right) \sin \left(\phi_{k}^{\mathrm{BF}}\right) \\
\cos \left(\phi_{k}^{\mathrm{BF}}\right)
\end{array}\right),
$$

where it is used explicitly that $\theta_{1}^{\mathrm{BF}}, \phi_{1}^{\mathrm{BF}}$ and $\phi_{2}^{\mathrm{BF}}$ are equal to zero. Thus, the set of $M=$ $3 N-6$ PsCs consist of $N-1$ vector lengths $R_{k}, N-2$ valence angles $\theta_{k}^{\mathrm{BF}}$ between the internal vectors $\mathbf{R}_{1}^{\mathrm{BF}}$ and $\mathbf{R}_{k}^{\mathrm{BF}}$ and $N-3$ dihedral angles $\phi_{k}^{\mathrm{BF}}=\phi_{2}^{\mathrm{E}_{2}}-\phi_{k>2}^{\mathrm{E}_{2}} \in[0,2 \pi]$ between the internal vectors $\mathbf{R}_{2}^{\mathrm{BF}}$ and $\mathbf{R}_{k}^{\mathrm{BF}}$ around $\mathbf{R}_{1}^{\mathrm{BF}}{ }^{81}$ Moreover, the $\mathrm{SF}$ and $\mathrm{BF}$ components of a given internal vector can be related trough the directional cosines matrix ${ }^{1,88}$ as

$$
\mathbf{R}_{k}^{\mathrm{SF}}=\boldsymbol{\Phi}(\boldsymbol{\Theta}) \mathbf{R}_{k}^{\mathrm{BF}}
$$

The nuclear KEO in PsCs can be obtained by inserting the momentum operator expressions, in terms of the radial and angular components shown in eqs. (16a) and (16b), into the expression given in eq. (15), along with the relation between SF and BF components of the internal vectors, as seen in eq. (18). Since the dot product $\hat{\mathbf{p}}_{k}^{\dagger} \cdot \hat{\mathbf{p}}_{l}$ does not depend on the frame of reference, unto which the momentum operators are projected, it is easier to consider the projection of these unto the BF frame of reference. This can be achieved by means of the unit vectors given in eq. (17). ${ }^{88}$ The nuclear KEO, including the deformational, Coriolis coupling and rotational contributions to the kinetic energy is then given by the analytical expression

$$
\begin{aligned}
& \hat{T}_{\text {nuc }}=-\frac{\hbar^{2}}{2} \sum_{k, l=1}^{N-1} \tilde{M}^{k l}\left(\hat{p}_{R_{k}}^{\dagger} \mathbf{e}_{k}^{\mathrm{BF}} \cdot \mathbf{e}_{l}^{\mathrm{BF}} \hat{p}_{R_{l}}-\hat{p}_{R_{k}}^{\dagger} \frac{\mathbf{e}_{k}^{\mathrm{BF}} \cdot\left(\mathbf{e}_{l}^{\mathrm{BF}} \times \hat{\mathbf{L}}_{k^{l}}^{\mathrm{BF}}\right)}{R_{l}}\right. \\
& \left.+\frac{\left(\left(\hat{\mathbf{L}}_{k}^{\mathrm{BF}}\right)^{\dagger} \times \mathbf{e}_{k}^{\mathrm{BF}}\right) \cdot \mathbf{e}_{l}^{\mathrm{BF}}}{R_{k}} \hat{p}_{R_{l}}-\frac{\left(\left(\hat{\mathbf{L}}_{k}^{\mathrm{BF}}\right)^{\dagger} \times \mathbf{e}_{k}^{\mathrm{BF}}\right) \cdot\left(\mathbf{e}_{l}^{\mathrm{BF}} \times \hat{\mathbf{L}}_{l}^{\mathrm{BF}}\right)}{R_{k} R_{l}}\right) .
\end{aligned}
$$


The particular expressions for the angular momentum operators depend on whether or not the associated internal vector $\mathbf{R}_{k}^{\mathrm{BF}}$ is used in defining the BF frame of reference. ${ }^{86}$ The precise expressions can be found in refs. 1 and 88. Inserting these expressions for the angular momentum operators projected unto the BF frame of reference into eq. (19), leads to the analytical expression for the nuclear KEO in PsCs. It is, subsequently, possible to generalize the expressions for deformational, Coriolis coupling and rotational kinetic energy contributions, which results in the overall form for the nuclear KEO in PsCs of

$$
\begin{aligned}
\hat{T}_{\mathrm{nuc}}=\frac{1}{2} & \sum_{m, n=1}^{M} \hat{p}_{m}^{\dagger} \Sigma^{m n}(\tilde{\mathbf{Q}}) \hat{p}_{n} \\
& +\frac{1}{2} \sum_{m=1}^{M} \sum_{\alpha=x, y, z} \hat{p}_{m}^{\dagger} \Gamma^{m \alpha}(\tilde{\mathbf{Q}}) \hat{J}_{\alpha}+\hat{J}_{\alpha} \Gamma^{\alpha m}(\tilde{\mathbf{Q}}) \hat{p}_{m} \\
& +\frac{1}{2} \sum_{\alpha, \beta=x, y, z} \hat{J}_{\alpha} \mu^{\alpha \beta}(\tilde{\mathbf{Q}}) \hat{J}_{\beta}+\hat{J}_{\beta} \mu^{\beta \alpha}(\tilde{\mathbf{Q}}) \hat{J}_{\alpha}
\end{aligned}
$$

The elements of the contravariant metric matrix $\mathbf{G}(\tilde{\mathbf{Q}})$ are for the polyspherical approach, given in terms of the PsCs $\tilde{\mathbf{Q}}$ and elements of the $\tilde{\mathbf{M}}$ matrix. The deformational, Coriolis coupling and rotational terms of the contravariant metric matrix are denoted as $\boldsymbol{\Sigma}(\tilde{\mathbf{Q}})$, $\boldsymbol{\Gamma}(\tilde{\mathbf{Q}})$ and $\boldsymbol{\mu}(\tilde{\mathbf{Q}})$ respectively. General expressions are provided by Gatti and Iung, ${ }^{1}$ for both orthogonal and non-orthogonal coordinates.

The molecular system can be approximated to be exclusively in the rotational ground state, by setting the total angular momentum operators $\hat{\mathbf{J}}$ to zero. This approximation entails that only the deformational part of the nuclear KEO is to be accounted for

$$
\hat{T}_{\mathrm{nuc}}=\frac{1}{2} \sum_{m, n=1}^{M} \hat{p}_{m}^{\dagger} \Sigma^{m n}(\tilde{\mathbf{Q}}) \hat{p}_{n} .
$$

Expanding this expression for the nuclear KEO in terms of the momentum operators conjugated to the internal curvilinear coordinates $\tilde{\mathbf{Q}}$ and invoking the chain rule, leads to the more practical form seen in eq. (2). ${ }^{57}$ 
It should be remarked that it is common practice, when working with curvilinear coordinates, to change from the Euclidean normalization convention to one that makes solving the TISE more convenient, e.g. Wilson normalization. ${ }^{3}$ Adopting a non-Euclidean normalization means that the Jacobian matrix determinant $\tilde{J}(\tilde{\mathbf{Q}})$, where the elements of the Jacobian matrix are the first derivatives of the Cartesian coordinates with respect to the polyspherical ones, is no longer used to define the volume element of integration. Instead, a positive and real valued function $\tilde{\rho}(\tilde{\mathbf{Q}})$ is substituted. This does, however, introduce an extra-potential

$$
\mathcal{V}_{\text {nuc }}(\tilde{\mathbf{Q}})=\tilde{J}^{\frac{1}{2}}(\tilde{\mathbf{Q}}) \tilde{\rho}^{-\frac{1}{2}}(\tilde{\mathbf{Q}})\left(\hat{T}_{\text {nuc }}^{[\tilde{J} \tilde{\mathbf{Q}})]} \tilde{\rho}^{\frac{1}{2}}(\tilde{\mathbf{Q}}) \tilde{J}^{-\frac{1}{2}}(\tilde{\mathbf{Q}})\right)
$$

as an additional multiplicative, i.e. non-differential term to the nuclear $\operatorname{KEO} \hat{T}_{\text {nuc }}^{[\tilde{J}(\tilde{\mathbf{Q}})]}$ of eq. (20). ${ }^{65,148}$ The nuclear KEO, to be used in solving the nuclear Schrödinger equation in non-Euclidean normalization, will therefore have the overall form

$$
\hat{T}_{\text {nuc }}^{[\tilde{\rho}(\tilde{\mathbf{Q}})]}=\hat{T}_{\text {nuc }}^{[\tilde{J}(\tilde{\mathbf{Q}})=\tilde{\rho}(\tilde{\mathbf{Q}})]}+\mathcal{V}_{\text {nuc }}(\tilde{\mathbf{Q}}) .
$$

Note that the Euclidean and Wilson normalization conventions corresponds to the particular choices $\tilde{\rho}(\tilde{\mathbf{Q}})=\tilde{J}(\tilde{\mathbf{Q}})$ and $\tilde{\rho}(\tilde{\mathbf{Q}})=1$ respectively.

\section{Polyspherical Displacement Coordinates}

The displacements, as found in eq. (9), are given in terms of $\tilde{\mathbf{d}}_{k^{\mathrm{m}_{k}}}^{m}=\eta_{k^{\mathrm{m}_{k}}}^{m} \tilde{\Lambda}_{m}$. The fractional displacements $\eta_{k^{\mathbf{m}_{k}}}^{m} \in[-1,1]$ belong to the set of rational numbers, while the scale factors 
$\tilde{\Lambda}_{m}$ can take different values depending on the type of internal coordinate

$$
\begin{aligned}
& \tilde{\Lambda}_{m}=\left\{\begin{array}{l}
\left(\tilde{\Lambda}_{m}\right)_{L}=\tilde{Q}_{m}^{\mathrm{ref}}-\sigma_{R_{k}} \\
\left(\tilde{\Lambda}_{m}\right)_{R}=R_{k}^{\mathrm{max}}-\sigma_{R_{k}}
\end{array}\right\} \text { if } \tilde{Q}_{m}=R_{k}, \\
& \tilde{\Lambda}_{m}=\left\{\begin{array}{l}
\left(\tilde{\Lambda}_{m}\right)_{L}=\tilde{Q}_{m}^{\mathrm{ref}}-\sigma^{\theta_{k}^{\mathrm{BF}}} \\
\left(\tilde{\Lambda}_{m}\right)_{R}=\left(\pi-\tilde{Q}_{m}^{\mathrm{ref}}\right)-\sigma_{k}^{\theta_{k}^{\mathrm{BF}}}
\end{array}\right\} \text { if } \tilde{Q}_{m}=\theta_{k}^{\mathrm{BF}}, \\
& \tilde{\Lambda}_{m}=\left\{\begin{array}{l}
\left(\tilde{\Lambda}_{m}\right)_{L}=\tilde{Q}_{m}^{\mathrm{ref}}-\sigma^{\phi_{k}^{\mathrm{BF}}} \\
\left(\tilde{\Lambda}_{m}\right)_{R}=\left(2 \pi-\tilde{Q}_{m}^{\mathrm{ref}}\right)-\sigma^{\phi_{k}^{\mathrm{BF}}}
\end{array}\right\} \text { if } \tilde{Q}_{m}=\phi_{k}^{\mathrm{BF}} .
\end{aligned}
$$

These scale factors indicate that the PES is shifted, so that $\tilde{\mathbf{Q}}^{\text {ref }}$ will be situated at the origin of the expansion but still comply with the correct periodicity of the associated curvilinear coordinates. The $\sigma^{\tilde{Q}_{m}}$ values ensure that the ADGA cannot displace the nuclear geometry past the natural periodicity of the internal coordinates, while the $R_{k}^{\max }$ value represents the maximum allowed displacement for distances. The initial boundary points for each mode are placed at the same fractional positions, as previously described for rectilinear coordinates, but there is no intentional link to the classical turning points of a harmonic oscillator, when using curvilinear coordinates in the ADGA.

\section{References}

(1) Gatti, F.; Iung, C. Exact and Constrained Kinetic Energy Operators for Polyatomic Molecules: The Polyspherical Approach. Phys. Rep. 2009, 484, 1-69.

(2) Sørensen, G. O. A New Approach to the Hamiltonian of Nonrigid Molecules. Top. Curr. Chem. 1979, 82, 97-175.

(3) Wilson, E. B.; Decius, J. C.; Cross, P. C. Molecular Vibrations, 1st ed.; Dover Publications Inc., 1980. 
(4) Jung, J. O.; Gerber, R. B. Vibrational Wave Functions and Energy Levels of Large Anharmonic Clusters: A Vibrational SCF Study of (Ar) ${ }_{13}$. J. Chem. Phys. 1996, 105, 10682.

(5) Kauppi, E. Ab Initio-Discrete Variable Representation Calculation of Vibrational Energy Levels. J. Chem. Phys. 1996, 105, 7986.

(6) Roitberg, A. E.; Gerber, R. B.; Ratner, M. A. A Vibrational Eigenfunction of a Protein: Anharmonic Coupled-Mode Ground and Fundamental Excited States of BPTI. J. Phys. Chem. B 1997, 101, 1700-1706.

(7) Carter, S.; Culik, S. J.; Bowman, J. M. Vibrational Self-Consistent Field Method for Many-Mode Systems: A New Approach and Application to the Vibrations of CO Adsorbed on $\mathrm{Cu}(100)$. J. Chem. Phys 1997, 107, 10458.

(8) Carter, S.; Bowman, J. M.; Harding, L. B. Ab Initio Calculations of Force Fields for $\mathrm{H}_{2} \mathrm{CN}$ and $\mathrm{ClHCN}$ and Vibrational Energies of $\mathrm{H}_{2}$ CN. Spectrochim. Acta Mol. Biomol. Spectrosc. 1997, 53, 1179-1188.

(9) Carter, S.; Bowman, J. M.; Handy, N. C. Extensions and Tests of MULTIMODE: A Code to Obtain Accurate Vibration/Rotation Energies of Many-Mode Molecules. Theor. Chem. Acc. 1998, 100, 191-198.

(10) Carter, S.; Bowman, J. M. The Adiabatic Rotation Approximation for Rovibrational Energies of Many-Mode Systems: Description and Tests of the Method. J. Chem. Phys. 1998, 108, 4397-4404.

(11) Chaban, G. M.; Jung, J. O.; Gerber, R. B. Ab Initio Calculation of Anharmonic Vibrational States of Polyatomic Systems: Electronic Structure Combined with Vibrational Self-Consistent Field. J. Chem. Phys. 1999, 111, 1823. 
(12) Yagi, K.; Taketsugu, T.; Hirao, K.; Gordon, M. S. Direct Vibrational Self-Consistent Field Method: Applications to $\mathrm{H}_{2} \mathrm{O}$ and $\mathrm{H}_{2}$ CO. J. Chem. Phys. 2000, 113, 1005.

(13) Bowman, J. M.; Carter, S.; Huang, X. MULTIMODE: A Code to Calculate Rovibrational Energies of Polyatomic Molecules. Int. Rev. Phys. Chem. 2003, 2, 533-549.

(14) Rauhut, G. Efficient Calculation of Potential Energy Surfaces for the Generation of Vibrational Wave Functions. J. Chem. Phys. 2004, 121, 9313.

(15) Christiansen, O. Vibrational Structure Theory: New Vibrational Wave Function Methods for Calculation of Anharmonic Vibrational Energies and Vibrational Contributions to Molecular Properties. Phys. Chem. Chem. Phys. 2007, 9, 2942-2953.

(16) Thompson, T. C.; Truhlar, D. G. Optimization of Vibrational Coordinates, with an Application to the Water Molecule. J. Chem. Phys. 1982, r7, 3031-3035.

(17) Moiseyev, N. On the SCF Method for Coupled-Vibron Systems. Chem. Phys. Lett. 1983, 98, 233-238.

(18) Yagi, K.; Keçeli, M.; Hirata, S. Optimized Coordinates for Anharmonic Vibrational Structure Theories. J. Chem. Phys. 2012, 13\%, 204118.

(19) Bowman, J. M. Self-Consistent Field Energies and Wave Functions for Coupled Oscillators. J. Chem. Phys. 1978, 68, 608-610.

(20) Gerber, R. B.; Ratner, M. A. A Semiclassical Self-Consistent Field (SC SCF) Approximation for Eigenvalues of Coupled-Vibration Systems. Chem. Phys. Lett. 1978, 68, $195-198$.

(21) Bowman, J. M. The Self-Consistent-Field Approach to Polyatomic Vibrations. Acc. Chem. Res. 1986, 19, 202-208.

(22) Christiansen, O. A Second Quantization Formulation of Multimode Dynamics. J. Chem. Phys. 2004, 120, 2140-2148. 
(23) Hansen, M. B.; Sparta, M.; Seidler, P.; Toffoli, D.; Christiansen, O. New Formulation and Implementation of Vibrational Self-Consistent Field Theory. J. Chem. Theory Comput. 2010, 6, 235-248.

(24) Yagi, K.; Otaki, H. Vibrational Quasi-Degenerate Perturbation Theory with Optimized Coordinates: Applications to Ethylene and Trans-1,3-butadiene. J. Chem. Phys. 2014, 140, 084113 .

(25) Thomsen, B.; Yagi, K.; Christiansen, O. Optimized Coordinates in Vibrational Coupled Cluster Calculations. J. Chem. Phys. 2014, 140, 154102.

(26) Thomsen, B.; Yagi, K.; Christiansen, O. A Simple State-Average Procedure Determining Optimal Coordinates for Anharmonic Vibrational Calculations. Chem. Phys. Lett. 2014, 610-611, 288-297.

(27) Yagi, K.; Thomsen, B. Infrared Spectra of Protonated Water Clusters, $\mathrm{H}^{+}\left(\mathrm{H}_{2} \mathrm{O}\right)_{4}$, in Eigen and Zundel Forms Studied by Vibrational Quasi-Degenerate Perturbation Theory. J. Phys. Chem. A 2017, 121, 2386-2398.

(28) Battocchio, G.; Madsen, N. K.; Christiansen, O. Density Matrices and Iterative Natural Modals in Vibrational Structure Theory. Mol. Phys. 2017, 115, 228-240.

(29) Madsen, N. K.; Godtliebsen, I. H.; Christiansen, O. Efficient Algorithms for Solving the Non-Linear Vibrational Coupled-Cluster Equations Using Full and Decomposed Tensors. J. Chem. Phys. 2017, 146, 134110.

(30) Rauhut, G. Configuration Selection as a Route Towards Efficient Vibrational Configuration Interaction Calculations. J. Chem. Phys. 2007, 127, 184109.

(31) Jacob, C. R.; Reiher, M. Localizing Normal Modes in Large Molecules. J. Chem. Phys. 2009, 130, 084106. 
(32) Jacob, C. R.; Luber, S.; Reiher, M. Analysis of Secondary Structure Effects on the IR and Raman Spectra of Polypeptides in Terms of Localized Vibrations. J. Phys. Chem. B 2009, 113, 6558-6573.

(33) Jacob, C. R. Theoretical Study of the Raman Optical Activity Spectra of 3 10-Helical Polypeptides. Chem. Phys. Chem. 2011, 12, 3291-3306.

(34) Panek, P. T.; Jacob, C. R. Efficient Calculation of Anharmonic Vibrational Spectra of Large Molecules with Localized Modes. Chem. Phys. Chem. 2014, 15, 3365-3377.

(35) Panek, P. T.; Jacob, C. R. Anharmonic Theoretical Vibrational Spectroscopy of Polypeptides. J. Phys. Chem. Lett. 2016, 7, 3084-3090.

(36) Cheng, X.; Steele, R. P. Efficient Anharmonic Vibrational Spectroscopy for Large Molecules Using Local-Mode Coordinates. J. Chem. Phys. 2014, 141, 104105.

(37) Cheng, X.; Talbot, J. J.; Steele, R. P. Tuning Vibrational Mode Localization with Frequency Windowing. J. Chem. Phys. 2016, 145, 124112.

(38) Ziegler, B.; Rauhut, G. Rigorous Use of Symmetry within the Construction of Multidimensional Potential Energy Surfaces. J. Phys. Chem. 2018, 149, 164110.

(39) Ziegler, B.; Rauhut, G. Localized Normal Coordinates in Accurate Vibrational Structure Calculations: Benchmarks for Small Molecules. J. Chem. Theory Comput. 2019, $15,4187-4196$.

(40) Panek, P. T.; Hoeske, A. A.; Jacob, C. R. On the Choice of Coordinates in Anharmonic Theoretical Vibrational Spectroscopy: Harmonic vs. Anharmonic Coupling in Vibrational Configuration Interaction. J. Chem. Phys. 2019, 150, 054107.

(41) Klinting, E. L.; König, C.; Christiansen, O. Hybrid Optimized and Localized Vibrational Coordinates. J. Phys. Chem. A 2015, 119, 11007-11021. 
(42) Klinting, E. L.; Thomsen, B.; Godtliebsen, I. H.; Christiansen, O. Employing General Fit-Bases for Construction of Potential Energy Surfaces with an Adaptive DensityGuided Approach. J. Chem. Phys. 2018, 148, 064113.

(43) König, C.; Hansen, M. B.; Godtliebsen, I. H.; Christiansen, O. FALCON: A Method for Flexible Adaptation of Local Coordinates of Nuclei. J. Chem. Phys. 2016, 144 , 074108 .

(44) Wang, Y.; Bowman, J. M. Towards an ab Initio Flexible Potential for Water, and Post-Harmonic Quantum Vibrational Analysis of Water Clusters. Chem. Phys. Lett. 2010, 491, 1-10.

(45) Wang, Y.; Bowman, J. M. Coupled-Monomers in Molecular Assemblies: Theory and Application to the Water Tetramer, Pentamer, and Ring Hexamer. J. Chem. Phys. 2012, 136, 144113.

(46) Mancini, J. S.; Bowman, J. M. On-the-fly Ab Intito Calculations of Anharmonic Vibrational Frequencies: Local-Monomer Theory and Application to HCl Clusters. J. Chem. Phys. 2013, 139, 164115.

(47) Liu, H.; Wang, Y.; Bowman, J. M. Transferable ab Initio Dipole Moment for Water: Three Applications to Bulk Water. J. Phys. Chem. B 2016, 120, 1735-1742.

(48) Zimmerman, P. M.; Smereka, P. Optimizing Vibrational Coordinates To Modulate Intermode Coupling. J. Chem. Theory Comput. 2016, 12, 1883-1891.

(49) Molina, A.; Smereka, P.; Zimmerman, P. M. Exploring the Relationship between Vibrational Mode Locality and Coupling Using Constrained Optimization. J. Chem. Phys. 2016, 144, 124111.

(50) Hanson-Heine, M. W. D. Examining the Impact of Harmonic Correlation on Vibra- 
tional Frequencies Calculated in Localized Coordinates. J. Chem. Phys. 2015, 143, 164104.

(51) Hanson-Heine, M. W. D. Reduced Basis Set Dependence in Anharmonic Frequency Calculations Involving Localized Coordinates. J. Chem. Theory Comput. 2018, 14, $1277-1285$.

(52) Nauts, A.; Chapuisat, X. Momentum, Quasi-momentum and Hamiltonian Operators in Terms of Arbitrary Curvilinear Coordinates, with Special Emphasis on Molecular Hamiltonians. Mol. Phys. 1985, 55, 1287-1318.

(53) Harthcock, M. A.; Laane, J. Calculation of Kinetic Energy Terms for the Vibrational Hamiltonian: Application to Large-Amplitude Vibrations Using One-, Two-, and Three-Dimensional Models. J. Mol. Spectrosc. 1982, 91, 300-324.

(54) Laane, J.; Harthcock, M. A.; Killough, P. M.; Bauman, L. E.; Cooke, J. M. Vector Representation of Large-Amplitude Vibrations for the Determination of Kinetic Energy Functions. J. Mol. Spectrosc. 1998, 91, 286-299.

(55) Senent, M. L. Determination of the Kinetic Energy Parameters of Non-Rigid Molecules. Chem. Phys. Lett. 1998, 296, 299-306.

(56) Luckhaus, D. 6D Vibrational Quantum Dynamics: Generalized Coordinate Discrete Variable Representation and (A)Diabatic Contraction. J. Chem. Phys. 2000, 113, $1329-1347$.

(57) Lauvergnat, D.; Nauts, A. Exact Numerical Computation of a Kinetic Energy Operator in Curvilinear Coordinates. J. Chem. Phys. 2002, 116, 8560.

(58) Blasco, S.; Lauvergnat, D. Quantum Study of the Internal Rotation of Methanol in Full Dimensionality $(1+11 \mathrm{D})$ : A Harmonic Adiabatic Approximation. Chem. Phys. Lett. 2003, 373, 344-349. 
(59) Mátyus, E.; Czakó, G.; Császár, A. G. Toward Black-Box-Type Full- and ReducedDimensional Variational (Ro)Vibrational Computations. J. Chem. Phys. 2009, 130, 134112.

(60) Lauvergnat, D.; Senent, M. L.; Jutier, L.; Hochlaf, M. Explicitly Correlated Treatment of $\mathrm{H}_{2} \mathrm{NSi}$ and $\mathrm{H}_{2} \mathrm{NSi}$ Radicals: Electronic Structure Calculations and Rovibrational Spectra. J. Chem. Phys. 2011, 135, 074301.

(61) Mackeprang, K.; Kjaergaard, H. G.; Salmi, T.; Hänninen, V.; Halonen, L. The Effect of Large Amplitude Motions on the Transition Frequency Redshift in Hydrogen Bonded Complexes: A Physical Picture. J. Chem. Phys. 2014, 140, 184309.

(62) Lauvergnat, D.; Nauts, A. Quantum Dynamics with Sparse Grids: A Combination of Smolyak Scheme and Cubature. Application to Methanol in Full Dimensionality. Spectrochim. Acta A 2014, 119, 18-25.

(63) Mackeprang, K.; Hänninen, V.; Halonen, L.; Kjaergaard, H. G. The Effect of Large Amplitude Motions on the Vibrational Intensities in Hydrogen Bonded Complexes. J. Chem. Phys. 2015, 142, 094304.

(64) Lauvergnat, D.; Luis, J. M.; Kirtman, B.; Reis, H.; Nauts, A. Numerical and Exact Kinetic Energy Operator using Eckart Conditions with one or Several Reference Geometries: Application to HONO. J. Chem. Phys. 2016, 144, 084116.

(65) Nauts, A.; Lauvergnat, D. Numerical on-the-fly Implementation of the Action of the Kinetic Energy Operator on a Vibrational Wave Function: Application to Methanol. Mol. Phys. 2018, 116, 3701-3709.

(66) Gatti, F.; Iung, C.; Menou, M.; Justum, Y.; Nauts, A.; Chapuisat, X. Vector Parametrization of the N-atom Problem in Quantum Mechanics I. Jacobi Vectors. J. Chem. Phys. 1998, 108, 8804-8820. 
(67) Gatti, F.; Iung, C.; Menou, M.; Chapuisat, X. Vector Parametrization of the N-atom Problem in Quantum Mechanics II. Coupled-Angular-Momentum Spectral Representations for Four-atom Systems. J. Chem. Phys. 1998, 108, 8821-8829.

(68) Gatti, F. Vector Parametrization of the N-atom Problem in Quantum Mechanics III. Separation into Two Subsystems: Application to $\mathrm{NH}_{3}$. J. Chem. Phys. 1999, 111, $7225-7235$.

(69) Leforestier, C.; Viel, A.; Gatti, F.; Muñoz, C.; Iung, C. The Jacobi-Wilson Method: A New Approach to the Description of Polyatomic Molecules. J. Chem. Phys. 2001, $114,2099$.

(70) Radau, R. Sur une Transformation des Équations Différentielles de la Dynamique. Ann. Sci. Éc. Norm. Supér. 1868, 5, 311-375.

(71) Smith, F. T. Modified Heliocentric Coordinates for Particle Dynamics. Phys. Rev. Lett. 1980, 45, 1157-1160.

(72) Wang, X.-G.; Carrington, T. Vibrational Energy Levels of $\mathrm{CH}_{5}^{+}$. J. Chem. Phys. 2008, 129, 234102.

(73) Bramley, M. J.; Handy, N. C. Efficient Calculation of Rovibrational Eigenstates of Sequentially Bonded Four-Atom Molecules. J. Chem. Phys. 1993, 98, 1378-1397.

(74) Johnson, B. R. On Hyperspherical Coordinates and Mapping the Internal Configurations of a Three Body System. J. Chem. Phys. 1980, 73, 5051-5058.

(75) Chapuisat, X.; Brunet, J.-P.; Nauts, A. Dynamics of Four Particles in Hyperspherical Coordinates. Chem. Phys. Lett. 1987, 136, 153-163.

(76) Horn, T. R.; Gerber, R. B.; Ratner, M. A. Vibrational States of Very Floppy Clusters: Approximate Separability and the Choice of Good Curvilinear Coordinates for $\mathrm{XeHe}_{2}$, I He. J. Chem. Phys. 1989, 91, 1813-1823. 
(77) Chapuisat, X.; Nauts, A.; Brunet, J.-P. Exact Quantum Molecular Hamiltonians I. Application to the Dynamics of Three Particles. Mol. Phys. 1991, 72, 1-31.

(78) Horn, T. R.; Gerber, R. B.; Valentini, J. J.; Ratner, M. A. Vibrational States and Structure of $\mathrm{Ar}_{3}$ : The Role of Three-Body Forces. J. Chem. Phys. 1991, 94, 67286736.

(79) Chapuisat, X. Exact Quantum Molecular Hamiltonians II. On the Choice of the Moving Frame of Reference. The Principal Axis System. Mol. Phys. 1991, 72, 1233-1265.

(80) Iung, C.; Gatti, F.; Viel, A.; Chapuisat, X. Vector Parametrization of the N-atom Problem in Quantum Mechanics with non-Orthogonal Coordinates. Phys. Chem. Chem. Phys. 1999, 1, 3377-3385.

(81) Chapuisat, X.; Iung, C. Vector Parametrization of the N-body Problem in Quantum Mechanics: Polyspherical Coordinates. Phys. Rev. A 1992, 45, 6217-6235.

(82) Mladenović, M. Rovibrational Hamiltonians for General Polyatomic Molecules in Spherical Polar Parametrization I. Orthogonal Representations. J. Chem. Phys. 2000, 112, 1070.

(83) Mladenović, M. Rovibrational Hamiltonians for General Polyatomic Molecules in Spherical Polar Parametrization II. Nonorthogonal Descriptions of Internal Molecular Geometry. J. Chem. Phys. 2000, 112, 1082.

(84) Gatti, F.; Muñoz, C.; Iung, C. A General Expression of the Exact Kinetic Energy Operator in Polyspherical Coordinates. J. Chem. Phys. 2001, 114, 8275-8281.

(85) Iung, C.; Gatti, F. Polyspherical Parametrization of an N-atom System: Principles and Applications. Int. J. Quant. Chem. 2006, 106, 130-151.

(86) Gatti, F.; Iung, C. Exact and Constrained Kinetic Energy Operators in Polyspherical Coordinates. J. Theor. Comput. Chem. 2003, 2, 507-522. 
(87) Gatti, F.; Nauts, A. Vector Parametrization, Partial Angular Momenta and Unusual Commutation Relations in Physics. Chem. Phys. 2003, 295, 167-174.

(88) Ndong, M.; Joubert-Doriol, L.; Meyer, H.-D.; Nauts, A.; Gatti, F.; Lauvergnat, D. Automatic Computer Procedure for Generating Exact and Analytical Kinetic Energy Operators Based on the Polyspherical Approach. J. Chem. Phys. 2012, 136, 034107.

(89) Sadri, K.; Lauvergnat, D.; Gatti, F.; Meyer, H.-D. Numeric Kinetic Energy Operators for Molecules in Polyspherical Coordinates. J. Chem. Phys. 2012, 136, 234112.

(90) Ndong, M.; Nauts, A.; Joubert-Doriol, L.; Meyer, H.-D.; Gatti, F.; Lauvergnat, D. Automatic Computer Procedure for Generating Exact and Analytical Kinetic Energy Operators Based on the Polyspherical Approach: General Formulation and Removal of Singularities. J. Chem. Phys. 2013, 139, 204107.

(91) Sadri, K.; Lauvergnat, D.; Gatti, F.; Meyer, H.-D. Rovibrational Spectroscopy Using a Kinetic Energy Eperator in Eckart Frame and the Multi-Configuration TimeDependent Hartree (MCTDH) Approach. J. Chem. Phys. 2014, 141, 234112.

(92) Christiansen, O. et al. MidAsCpP: Molecular interactions, dynamics and simulations Chemistry program package in C++. 2019; https://midascpp.gitlab.io/.

(93) Born, M.; Oppenheimer, R. Zur Quantentheorie der Molekeln. Ann. Phys. 1927, 389, $457-484$.

(94) Christiansen, O. Selected New Developments in Vibrational Structure Theory: Potential Construction and Vibrational Wave Function Calculations. Phys. Chem. Chem. Phys. 2012, 14, 6672-6687.

(95) Meyer, H.-D.; Gatti, F.; Worth, G. A. Multidimensional Quantum Dynamics, 1st ed.; Wiley, 2009. 
(96) Gatti, F.; Lasorne, B.; Meyer, H.-D.; Nauts, A. Applications of Quantum Dynamics in Chemistry, 3rd ed.; Springer, 2017.

(97) Vendrell, O.; Gatti, F.; Lauvergnat, D.; Meyer, H.-D. Full-Dimensional (15dimensional) Quantum-Dynamical Simulation of the Protonated Water Dimer I. Hamiltonian Setup and Analysis of the Ground Vibrational State. J. Chem. Phys. 2007, 127, 184302.

(98) Richter, F.; Thaunay, F.; Lauvergnat, D.; Carbonnière, P. Anharmonic Vibrational Treatment Exclusively in Curvilinear Valence Coordinates: The Case of Formamide. J. Phys. Chem. A 2015, 119, 11719-11728.

(99) Watson, J. K. G. Simplification of the Molecular Vibration-Rotation Hamiltonian. Mol. Phys. 1968, 15, 479-490.

(100) Kongsted, J.; Christiansen, O. Automatic Generation of Force Fields and Property Surfaces for Use in Variational Vibrational Calculations of Anharmonic Vibrational Energies and Zero-Point Vibrational Averaged Properties. J. Chem. Phys. 2006, 125, $124108-124123$.

(101) König, C.; Christiansen, O. Linear-Scaling Generation of Potential Energy Surfaces Using a Double Incremental Expansion. J. Chem. Phys. 2016, 145, 064105.

(102) Sparta, M.; Toffoli, D.; Christiansen, O. An Adaptive Density-Guided Approach for the Generation of Potential Energy Surfaces of Polyatomic Molecules. Theor. Chem. Acc. 2009, 123, 413-429.

(103) Sparta, M.; Høyvik, I.-M.; Toffoli, D.; Christiansen, O. Potential Energy Surfaces for Vibrational Structure Calculations from a Multiresolution Adaptive Density-Guided Approach: Implementation and Test Calculations. J. Phys. Chem. A 2009, 113, 87128723. 
(104) Sparta, M.; Hansen, M. B.; Matito, E.; Toffoli, D.; Christiansen, O. Using Electronic Energy Derivative Information in Automated Potential Energy Surface Construction for Vibrational Calculations. J. Chem. Theory Comput. 2010, 6, 3162-3175.

(105) Schmitz, G.; Artiukhin, D. G.; Christiansen, O. Approximate High Mode Coupling Potentials using Gaussian Process Regression and Adaptive Density Guided Sampling. J. Chem. Phys. 2019, 150, 131102.

(106) Christiansen, O. Vibrational Coupled Cluster Theory. J. Chem. Phys. 2004, 120, 2149-2159.

(107) Györffy, W.; Seidler, P.; Christiansen, O. Solving the Eigenvalue Equations of Correlated Vibrational Structure Methods: Preconditioning and Targeting Strategies. J. Chem. Phys. 2009, 131, 024108.

(108) Seidler, P.; Christiansen, O. Automatic Derivation and Evaluation of Vibrational Coupled Cluster Theory Equations. J. Chem. Phys. 2009, 131, 234109.

(109) Bowman, J. M.; Christoffel, K.; Tobin, F. Application of SCF-SI Theory to Vibrational Motion in Polyatomic Molecules. J. Phys. Chem. 1979, 83, 905-912.

(110) Christoffel, K. M.; Bowman, J. M. Investigations of Self-Consistent Field, SCF CI and Virtual State Configuration Interaction Vibrational Energies for a Model Three-Mode System. Chem. Phys. Lett. 1982, 85, 220-224.

(111) Neff, M.; Rauhut, G. Toward Large Scale Vibrational Configuration Interaction Calculations. J. Chem. Phys. 2009, 131, 124129.

(112) Madsen, N. K.; Godtliebsen, I. H.; Losilla, S. A.; Christiansen, O. TensorDecomposed Vibrational Coupled-Cluster Theory: Enabling Large-Scale, Highly Accurate Vibrational-Structure Calculations. J. Chem. Phys. 2018, 148, 024103. 
(113) Christiansen, O. Response Theory for Vibrational Wave Functions. J. Chem. Phys. 2005, 122, 194105.

(114) Seidler, P.; Christiansen, O. Vibrational Excitation Energies from Vibrational Coupled Cluster Response Theory. J. Chem. Phys. 2007, 126, 204101.

(115) Zoccante, A.; Seidler, P.; Christiansen, O. Computation of Expectation Values from Vibrational Coupled-Cluster at the Two-Mode Coupling Level. J. Chem. Phys. 2011, $134,154101$.

(116) Seidler, P.; Sparta, M.; Christiansen, O. Vibrational Coupled Cluster Response Theory: A General Implementation. J. Chem. Phys. 2011, 134, 054119.

(117) Ahlrichs, R. et al. Turbomole: Program Package for ab Initio Electronic Structure Calculations. 2016; http://www.turbomole.com.

(118) Ahlrichs, R.; Bär, M.; Häser, M.; Horn, H.; Kölmel, C. Electronic Structure Calculations on Workstation Computers: The Program System Turbomole. Chem. Phys. Lett. 1989, 162-169, 165-169.

(119) Hättig, C.; Tew, D. P.; Köhn, A. Communications: Accurate and Efficient Approximations to Explicitly Correlated Coupled-Cluster Singles and Doubles, CCSD-F12. J. Chem. Phys. 2010, 132, 231102.

(120) Peterson, K. A.; Adler, T. B.; Werner, H.-J. Systematically Convergent Basis Sets for Explicitly Correlated Wave Functions: The Atoms H, He, B-Ne, and Al-Ar. J. Chem. Phys. 2008, 128, 084102.

(121) Dunning, T. H. Gaussian Basis Sets for Use in Correlated Molecular Calculations. I. The Atoms Boron Through Neon and Hydrogen. J. Chem. Phys. 1989, 90, 1007-1023.

(122) Toffoli, D.; Sparta, M.; Christiansen, O. Accurate Multimode Vibrational Calculations 
Using A B-spline Basis: Theory, Tests and Application to Dioxirane and Diazirinone. Mol. Phys. 2011, 109, 673-685.

(123) Toffoli, D.; Kongsted, J.; Christiansen, O. Automatic Generation of Potential Energy and Property Surfaces of Polyatomic Molecules in Normal Coordinates. J. Chem. Phys. 2007, 127, 204106.

(124) Nauts, A.; Chapuisat, X. Hamiltonians for Constrained N-particle Systems. Chem. Phys. Lett. 1987, 136, 164-170.

(125) Małyszek, P.; Koput, J. Accurate ab Initio Potential Energy Surface and VibrationRotation Energy Levels of Hydrogen Peroxide. J. Comput. Chem. 2013, 34, 337-345.

(126) Olson, W.; Hunt, R.; Young, B.; Maki, A.; Brault, J. Rotational Constants of the Lowest Torsional Component $(0 G)$ of the Ground State and Lowest Torsional Component $(1 G)$ of the First Excited Torsional State of Hydrogen Peroxide. J. Mol. Spectrosc. 1988, 127, 12-34.

(127) Flaud, J.; Camy-Peyret, C.; Johns, J. W. C.; Carli, B. The Far Infrared Spectrum of $\mathrm{H}_{2} \mathrm{O}_{2}$. First Observation of the Staggering of the Levels and Determination of the Cis Barrier. J. Chem. Phys. 1989, 91, 1504-1510.

(128) Perrin, A.; Flaud, J.-M.; Camy-Peyret, C.; Goldman, A.; Murcray, F.; Blatherwick, R. New Analysis of the $\nu_{6}$ Band of $\mathrm{H}_{2} \mathrm{O}_{2}$ : The $(n, \tau)=(0,1),(1,1),(2,1),(0,3)$, and $(1,3)$ Torsional Subbands. J. Mol. Spectrosc. 1990, 142, 129-147.

(129) Camy-Peyret, C.; Flaud, J.-M.; Johns, J.; Noël, M. Torsion-Vibration Interaction in $\mathrm{H}_{2} \mathrm{O}_{2}$ : First High-Resolution Observation of $\nu_{3}$. J. Mol. Spectrosc. 1992, 155, 84-104.

(130) Perrin, A.; Valentin, A.; Flaud, J.; Camypeyret, C.; Schriver, L.; Schriver, A.; Arcas, P. The 7.9- $\nu \mathrm{m}$ Band of Hydrogen Peroxide: Line Positions and Intensities. J. Mol. Spectrosc. 1995, 171, 358-373. 
(131) Klee, S.; Winnewisser, M.; Perrin, A.; Flaud, J.-M. Absolute Line Intensities for the $\nu_{6}$ Band of $\mathrm{H}_{2} \mathrm{O}_{2}$. J. Mol. Spectrosc. 1999, 195, 154-161.

(132) Koput, J.; Carter, S.; Handy, N. C. Potential Energy Surface and VibrationalRotational Energy Levels of Hydrogen Peroxide. J. Phys. Chem. A 1998, 102, 63256330.

(133) Chen, R.; Ma, G.; Guo, H. Full-Dimensional Quantum Calculation of the Vibrational Energy Levels of Hydrogen Peroxide (HOOH). Chem. Phys. Lett. 2000, 320, 567-574.

(134) Koput, J.; Carter, S.; Handy, N. C. Ab Initio Prediction of the Vibrational-Rotational Energy Levels of Hydrogen Peroxide and its Isotopomers. J. Chem. Phys. 2001, 115, $8345-8350$.

(135) Chen, R.; Ma, G.; Guo, H. Six-Dimensional Quantum Calculations of Highly Excited Vibrational Energy Levels of Hydrogen Peroxide and its Deuterated Isotopomers. J. Chem. Phys. 2001, 114, 4763-4774.

(136) Rauhut, G.; Knizia, G.; Werner, H.-J. Accurate Calculation of Vibrational Frequencies using Explicitly Correlated Coupled-Cluster Theory. J. Chem. Phys. 2009, 130, 054105 .

(137) Hollman, D. S.; Schaefer, H. F. In Search of the Next Holy Grail of Polyoxide Chemistry: Explicitly Correlated ab Initio Full Quartic Force Fields for HOOH, HOOOH, HOOOOH, and their Isotopologues. J. Chem. Phys. 2012, 136, 084302.

(138) Schoenberg, I. J. Contributions to the Problem of Approximation of Equidistant Data by Analytic Functions, Part A: On the Problem of Smoothing or Graduation, a First Class of Analytic Approximation Formulas. Quart. Appl. Math. 1946, 4, 45-99.

(139) Schoenberg, I. J. In On Spline Functions, 1st ed.; Shisha, O., Ed.; Academic Press, 1967; pp 255-291, with a supplement by T. N. E. Greville. 
(140) de Boor, C. A Practical Guide to Splines, 1st ed.; Springer, 1978; Vol. 27.

(141) Piegl, L.; Tiller, W. The NURBS Book, 2nd ed.; Springer, 1995.

(142) Bachau, H.; Cormier, E.; Decleva, P.; Hansen, J. E.; Martín, F. Applications of Bsplines in Atomic and Molecular Physics. Rep. Prog. Phys. 2001, 64, 1815-1942.

(143) Klinting, E. L.; Christiansen, O.; König, C. Toward Accurate Theoretical Vibrational Spectra: A Case Study for Maleimide. J. Chem. Phys. A 2020, 124, 2616-2627.

(144) Seidler, P.; Hansen, M. B.; Christiansen, O. Towards Fast Computations of Correlated Vibrational Wave Functions: Vibrational Coupled Cluster Response Excitation Energies at the Two-Mode Coupling Level. J. Chem. Phys. 2008, 128, 154113.

(145) Artiukhin, D. G.; Klinting, E. L.; König, C.; Christiansen, O. Adaptive DensityGuided Approach to Double Incremental Potential Energy Surface Construction. arXiv 2020, 2002.04919.

(146) Eckart, C. Some Studies Concerning Rotating Axes and Polyatomic Molecules. Phys. Rev. 1935, 47, 552-558.

(147) Sayvetz, A. The Kinetic Energy of Polyatomic Molecules. J. Chem. Phys. 1939, 7, 383.

(148) Chapuisat, X.; Belafhal, A.; Nauts, A. N-body Quantum-Mechanical Hamiltonians: Extrapotential Terms. J. Mol. Spectrosc. 1991, 149, 274-304. 


\section{TOC Graphic}

Mean Absolute Deviations in $\mathrm{cm}^{-1}$ for VCC $[n]$ from FVCI

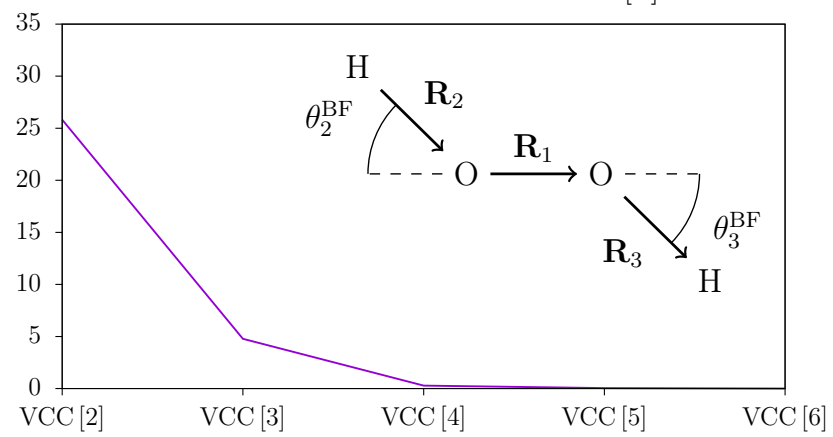

